\title{
Introduction of Planar Compliant Joints Designed for Combined Bending and Axial Loading Conditions in Lamina Emergent Mechanisms
}

Samuel E. Wilding

Brigham Young University - Provo

Larry L. Howell

Brigham Young University - Utah, Ihowell@byu.edu

Spencer P. Magleby

Follow this and additional works at: https://scholarsarchive.byu.edu/facpub

Part of the Mechanical Engineering Commons

\section{Original Publication Citation}

Wilding, S.E., Howell, L.L., Magleby, S.P., “Introduction of Planar Compliant Joints Designed for Compressive and Tensile Loading Conditions in Lamina Emergent Mechanisms," Mechanism and Machine Theory, Vol. 56, pp. 1-15, DOI: 10.1016/j.mechmachtheory.2012.05.007, 2012.

\section{BYU ScholarsArchive Citation}

Wilding, Samuel E.; Howell, Larry L.; and Magleby, Spencer P., "Introduction of Planar Compliant Joints Designed for Combined Bending and Axial Loading Conditions in Lamina Emergent Mechanisms" (2012). Faculty Publications. 1561.

https://scholarsarchive.byu.edu/facpub/1561

This Peer-Reviewed Article is brought to you for free and open access by BYU ScholarsArchive. It has been accepted for inclusion in Faculty Publications by an authorized administrator of BYU ScholarsArchive. For more information, please contact ellen_amatangelo@byu.edu. 


\title{
Introduction of Planar Compliant Joints Designed for Combined Bending and Axial Loading Conditions in Lamina Emergent Mechanisms
}

\author{
Samuel E. Wilding, Larry L. Howell*, Spencer P. Magleby \\ Brigham Young University, Department of Mechanical Engineering, Provo, UT 84602, United \\ States
}

\begin{abstract}
This work introduces three joints to allow motion in lamina emergent mechanisms (LEMs) that were designed to have minimal parasitic motion under tension, compression, and a combination of tension and compression loading. Closed-form models of the joints were developed and combined with optimization algorithms for maximum flexibility in bending and then modeled using finite element analysis (FEA). The FEA results were used to predict the stiffnesses of the joints in bending, tension, and compression. As a baseline, lamina emergent torsional (LET) joints were designed to match the bending stiffness of each of the joints, so that the tensile-compressive performance could be compared. The joints demonstrate improved off-axis stiffness in tensile and/or compressive stiffness, at the cost of limited bending flexibility compared to the LET joint and requiring more material area. The resulting joints provide a broad set of capabilities that can be used by designers in lamina emergent mechanism design.
\end{abstract}

Keywords: lamina emergent mechanisms, compliant mechanisms, compliant joints, tension, compression

\section{Introduction}

Lamina emergent mechanisms (LEMs) are compliant mechanisms that can be fabricated from planar sheets [1]. Upon actuation, LEMs "emerge" out of the fabrication plane. A preliminary study of LEM joints was performed by Winder et al. which

\footnotetext{
${ }^{*}$ Corresponding Author. Tel.: +1 8014228037.

Email address: lhowell@byu.edu (Larry L. Howell)
} 
discusses a method of using serial chain approximations to create LEM joints with the desired characteristics [2]. Multiple applications of LEMs have been proposed [3] ranging from disposable mechanisms to deployable mechanisms. Other emerging areas of application for the proposed joints, and for LEMs as a whole, include adaptive morphing structures and engineering applications of origami principles. An example of a LEM is shown in Figure 1.

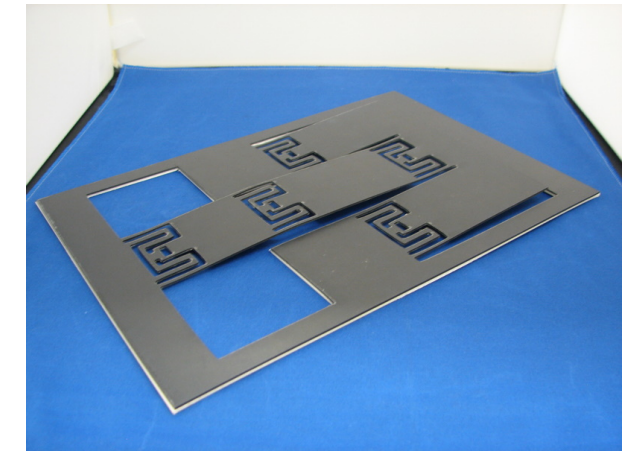

(a) 4R linkage in planar position

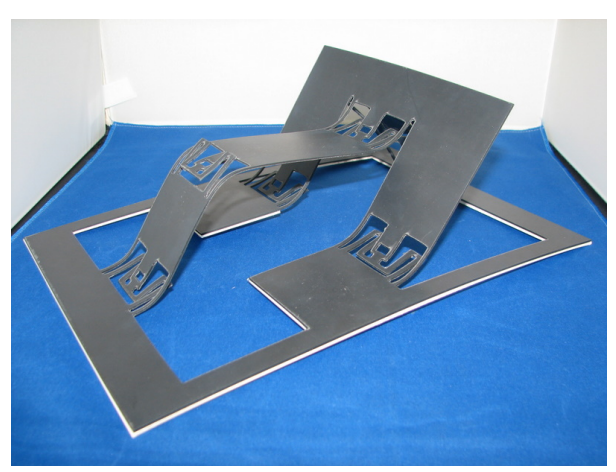

(b) 4R linkage in actuated position

Figure 1: 4R linkage with Inverted Tension Lamina Emergent Joints (IT-LEJ)

LEMs offer the advantages of a topology compatible with planar layers, inherent compactness, and compatibility with simple manufacturing processes in addition to the advantages currently possessed by compliant mechanisms. Also, many LEMs posses a monolithic structure. The advantages of a monolithic structure, as well as advantages of compliant mechanisms are discussed in [4]. Thorough documentation on the advantages and disadvantages of compliant mechanisms can be found in [5].

Lamina emergent torsional (LET) joints [6] have been finding use in LEMs; however, they do not lend themselves well to applications where the joints experience significant tension or compression loads because they allow considerable parasitic motion under those conditions. An example of designing for parasitic motion in LET joints is shown in [7], where minimum potential energy optimization is used to determine the position of a fully compliant mechanism, taking into account the parasitic motion of the joint.

This paper introduces three joints that are compatible with lamina emergent mechanisms and are intended for use under combined bending and axial loads. LET joints are used as a benchmark comparison for the joints. For each new joint, a LET joint was modeled to have the same bending stiffness and maximum axial (tensile and/or compressive) stiffness. The compressive and/or tensile stiffnesses of the joints 
were then compared. The new joints exhibit increased off-axis stiffness (reduced parasitic motion) compared to the LET joint when subjected to compressive and/or tensile loads.

\section{Background}

Designing LEMs can be challenging because all mechanism components are confined to the plane of fabrication. Joint design can be particularly challenging. Jacobson et al. [6] developed the lamina emergent torsional (LET) joint; a LEM compatible joint that allows for large rotations in situations where off-axis stiffness is not critical. Off-axis stiffness is defined as the stiffness in any direction other than the desired motion of the joint [8], and greater off-axis stiffness results in reduced parasitic motion. Ferrell et al. [9] describe LEM joints with specific application to metals. Other work in compliant joints, while not restricted to LEMs, addresses many of the challenges of compliant joints. For example, Trease et al. discuss large-displacement compliant joints [8]. Yao et al. kinematically describe the folding of cartons where creases act as joints [10]. Zhao et al. discuss a monolithic flexural pivot [11]. Pei et al. discuss the design of cartwheel flexural hinges [12]. Dai and Jones discuss the modeling of metamorphic structures that begin as flat sheets and through folding become a structure [13]. Other examples of compliant joints include applications in microrobotics [14, 15], spherical mechanisms [16, 17], and precision devices [18, 19, 20, 21].

Under some loading conditions, lamina emergent joints will be subjected to nonideal loading conditions, resulting in tension or compression loads on the joints. The most common lamina emergent joints, such as the LET joint, are not well suited for this loading condition. The extreme geometric limitations inherent in lamina emergent mechanisms make it difficult to create joints with the desired behaviors. Because of these and other conditions, it would be helpful to have an increased choice of lamina emergent joints that would be flexible under bending loads but stiffer under axial loads.

\subsection{Isolation and Inversion}

Compression of compliant joints is often a concern and many compliant joints are not able to adequately support compressive loads. Guérinot et al. [22] describe two methods, isolation and inversion, for increasing the amount of compressive load a compliant joint can carry. Isolation is accomplished by diverting the compressive load from the flexible segment(s) to a rigid element(s) of the joint. An example is a passive rest that comes into contact when the joint is put in compression. Isolation is often a challenge with LEMs. Because the joint is restricted to a plane, areas that 
come into contact are small, making the passive rests unstable. More rests can be added, but they will be coplanar for a single layer LEM. Inversion is more applicable to LEMs. Inversion involves reconfiguring the joint so that the members prone to buckling are put in tension when the joint is compressed.

\subsection{The Lamina Emergent Torsional (LET) Joint}

The LET joint, shown in Figure 2(a), will be used as a benchmark for comparison of the new joints. The LET joint described here is assumed to be symmetric about the $x$ and $y$ axes. The subscripts of the $k$ labels in Figure 2(a) indicate the primary deflection mode of the labeled member when the joint is deflected about the $y$ axis: $b$ represents bending and $t$ represents torsion. A linear spring model is shown in 2(b). This model will be used to determine a closed-form model of the stiffness in bending about the $y$ axis. The dimension labels used for the stiffness derivation and throughout this work for the LET joint are shown in Figure 2(a).

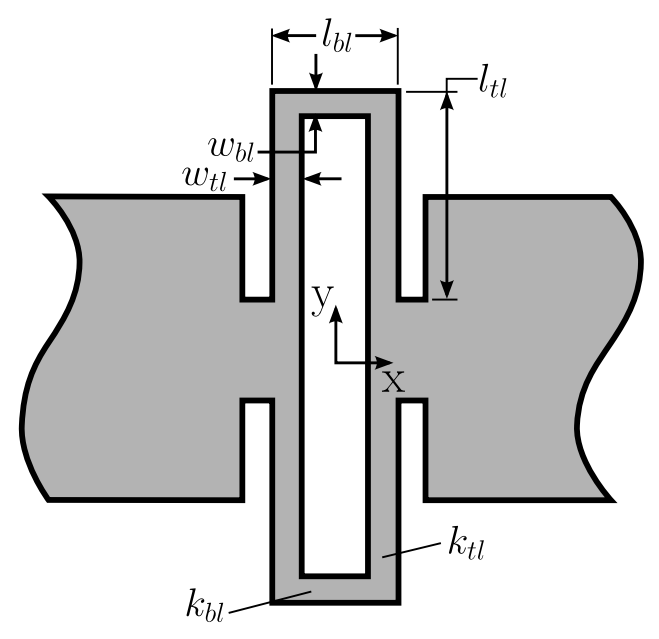

(a) The LET joint

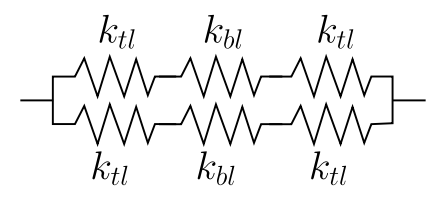

(b) Spring model of the LET joint

Figure 2: Lamina Emergent Torsional (LET) joint with dimension labels and its associated spring model

As developed in [6], the bending stiffness of the LET joint can be derived as

$$
k_{e q, b e n d}=\frac{2 k_{t l} k_{b l}}{k_{t l}+2 k_{b l}}
$$


where

$$
k_{b l}=\frac{E w_{b l} t^{3}}{12 l_{b l}}
$$

and

$$
k_{t l}=w_{t l} t^{3} \frac{G}{l_{t l}}\left[\frac{1}{3}-0.21 \frac{t}{w_{t l}}\left(1-\frac{t^{4}}{12 w_{t l}}\right)\right]
$$

In Equations (1) through (3), $t$ represents the thickness of the material, $E$ represents the modulus of elasticity, and $G$ represents the modulus of rigidity. Equation (3) is used to describe a rectangular beam in torsion [23]. For all the analyses in this work, the material was assumed to be polypropylene; with $E=1.4 G P a, \nu=0.42$, and $G=0.493 G P a$.

This model provides a valuable estimate of the joints' stiffness in bending, which is particularly important for this study because the joints are intended to have low stiffness under bending loads and higher stiffness under axial loads as compared to existing lamina emergent joints. The moment load is used here because it is a consistent reflection of how joints are implemented in lamina emergent mechanisms. Other analytical models are provided to evaluate the other extreme of axial loads. Assuming the bending segments of the joint are considered to be completely rigid in tension and compression (along the $x$ axis), i.e. $k_{b l}=\infty$, and assuming small deflections, the compressive $\left(k_{e q, c o m p}\right)$ and tensile $\left(k_{e q, \text { tens }}\right)$ stiffnesses of the joint can be modeled as

$$
k_{e q, \text { comp }}=k_{e q, t e n s}=\frac{E t w_{t l}^{3}}{l_{t l}^{3}}
$$

This model is valid for small deflections and assumes the bending segments of the joint can be modeled as rigid. This model is particularly useful when combined with an optimization algorithm, as discussed later. When this simplified model is inadequate, nonlinear finite element analysis will be used.

The LET joint is capable of large angular deflections and is geometrically simple, making it easy to implement in LEMs. The disadvantages of the LET joint include low off-axis stiffness and a potential for parasitic motion under tension and compression loading. Note that it is assumed that the tension and compression loading is completely in the $x y$ plane, as illustrated in Figure 3. In this paper the LET joint is used as a benchmark for the other joints.

The ideal lamina emergent joint will be small relative to the more rigid segments of the mechanism. When an actuation force is applied to the mechanism, it is transferred through these stiffer elements to the joints. Since the rigid links are intended to be much longer than the flexible joints, the moment arm associated with 


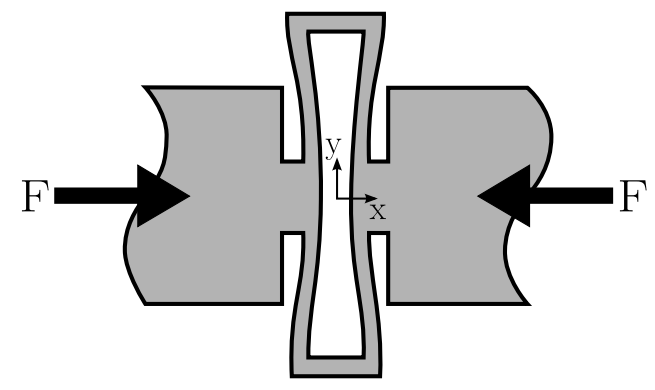

(a) LET joint loaded in compression

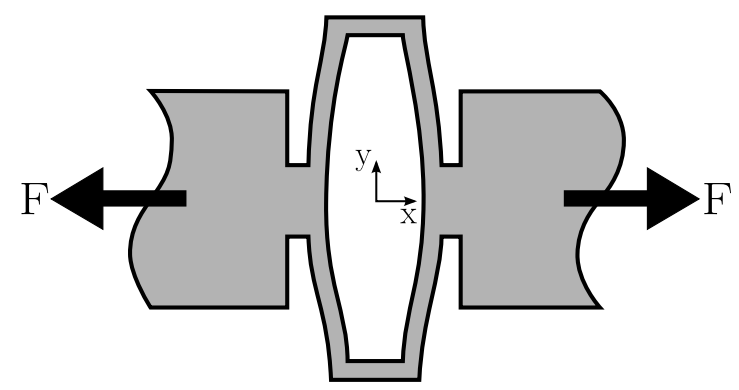

(b) LET joint loaded in tension

Figure 3: Examples of parasitic motion in the LET joint

the force tends to be large and a moment is the dominate load. This characteristic has allowed the LET joint to be a useful in LEMs in spite of its lacking high stiffness under axial loads. However, there are some conditions where the actuation load is near the joint or otherwise induces high axial loads in addition to bending loads. Multiple analytical models are provided for loadings under these different conditions.

\subsection{Method}

Two sets of models are used in this work: small-deflection, closed-form models and large-deflection finite element models. The closed-form models are used primarily as synthesis tools, whereas the large-deflection finite element models are used primarily as analysis tools to evaluate the joints behavior under large deflections. The closedform small-deflection equations serve several purposes: First, they made it possible to evaluate many different possibilities (not all of which are described in this paper) and to down-select to the configurations discussed here that are deserving of more in-depth study and application. Second, the models are readily optimized and the 
results are used as the basis for more in-depth study using nonlinear finite element analysis. Third, although they are primarily used as a synthesis tool, they can also be used as an analysis tool to provide an estimate of the motion for small deflections for a given configuration. The large-deflection finite element models were used primarily for analysis of pre-determined geometry defined using the analytical models. Nonlinear FEA provides a powerful tool for evaluating the performance of the joints under large-deflections that are outside the range of the analytical models. The FEA results also verify that the joints can be used in this range. For the design of joints undergoing large deflections, nonlinear finite element analysis would be an appropriate tool for their analysis. The FEA models were also used to verify the analytical models in the small-deflection range.

The following steps will be used in the development and evaluation of the new joints:

1. Develop a small-deflection, closed-form model of joint bending stiffness

2. Use the small-deflection, closed-form model to optimize joint geometry for minimum bending stiffness, assuming material properties and material thickness are constant

3. Create a large-deflection FEA model of the joint being studied using the dimensions from the previous step to obtain a more accurate bending stiffness value and to determine the compressive and/or tensile stiffness(es) value(s)

4. Optimize the benchmark LET joint geometry using Equations (1) through (4) to match the bending stiffness to that obtained in the previous step and maximize compressive and/or tensile stiffness(es)

5. Create an FEA model of the LET joint using the dimensions from the previous step to obtain a more accurate bending and compressive and/or tensile stiffness(es) value(s)

6. Compare stiffness results from FEA models to the benchmark LET joint and joint being studied

These steps were used for each of the three joints introduced in this paper. The LET joint is used as a benchmark of comparison to demonstrate the new joints' advantages and disadvantages. The resulting joints are useful in both small- and large-deflection applications.

\section{The Inverted Lamina Emergent Joint (I-LEJ)}

The Inverted Lamina Emergent Joint (I-LEJ) was designed using the principle of inversion [22] and is capable of bearing compressive loads with reduced parasitic 
motion compared with the LET joint. Its name comes from the fact that the long bending members (labeled $k_{b 2}$ in Figure $4(\mathrm{a})$ ) are put in tension when the joint is compressed along the $-x$ axis. This joint, with its corresponding spring model, is shown in Figure 4. Most of the movement of this joint comes from the bending of long, flexible segments, although some torsion is present in the torsion members aligned with the $y$ axis.

\subsection{Closed-Form Model}

A closed-form model of the joint's bending stiffness was developed for small deflections. Referring to the model shown in Figure 4(b) and the dimensions indicated in Figure 5, the bending stiffness $\left(k_{e q, b e n d}\right)$ of the joint was derived assuming small deflections and pure moment loading:

$$
k_{\text {eq,bend }}=\frac{2 k_{b 1} k_{b 2} k_{b 3} k_{b 4} k_{t 1} k_{t 2} k_{t 3}}{k_{\text {sum }} k_{b 4}+2 k_{b 1} k_{b 2} k_{b 3} k_{t 1} k_{t 2} k_{t 3}}
$$

where

$$
\begin{aligned}
k_{s u m}= & k_{b 1} k_{b 2} k_{b 3} k_{t 2} k_{t 3}+k_{b 1} k_{b 2} k_{b 3} k_{t 2} k_{t 3}+k_{b 1} k_{b 2} k_{b 3} k_{1 t} k_{t 3} \\
& +k_{b 1} k_{b 3} k_{t 1} k_{t 2} k_{t 3}+k_{b 1} k_{b 2} k_{b 3} k_{t 1} k_{t 2}+k_{b 1} k_{b 2} k_{t 1} k_{t 2} k_{t 3}
\end{aligned}
$$

and

$$
\begin{aligned}
& k_{b 1}=\frac{E w_{b 1} t^{3}}{12 l_{b 1}} \\
& k_{b 2}=\frac{E w_{b 2} t^{3}}{12 l_{b 2}} \\
& k_{b 3}=\frac{E w_{b 3} t^{3}}{12 l_{b 3}} \\
& k_{b 4}=\frac{E w_{b 4} t^{3}}{12 l_{b 4}} \\
& k_{t 1}=w_{t 1} t^{3} \frac{G}{l_{t 1}}\left[\frac{1}{3}-0.21 \frac{t}{w_{t 1}}\left(1-\frac{t^{4}}{12 w_{t 1}^{4}}\right)\right] \\
& k_{t 2}=w_{t 2} t^{3} \frac{G}{l_{t 2}}\left[\frac{1}{3}-0.21 \frac{t}{w_{t 2}}\left(1-\frac{t^{4}}{12 w_{t 2}^{4}}\right)\right] \\
& k_{t 3}=w_{t 3} t^{3} \frac{G}{l_{t 3}}\left[\frac{1}{3}-0.21 \frac{t}{w_{t 3}}\left(1-\frac{t^{4}}{12 w_{t 3}^{4}}\right)\right]
\end{aligned}
$$




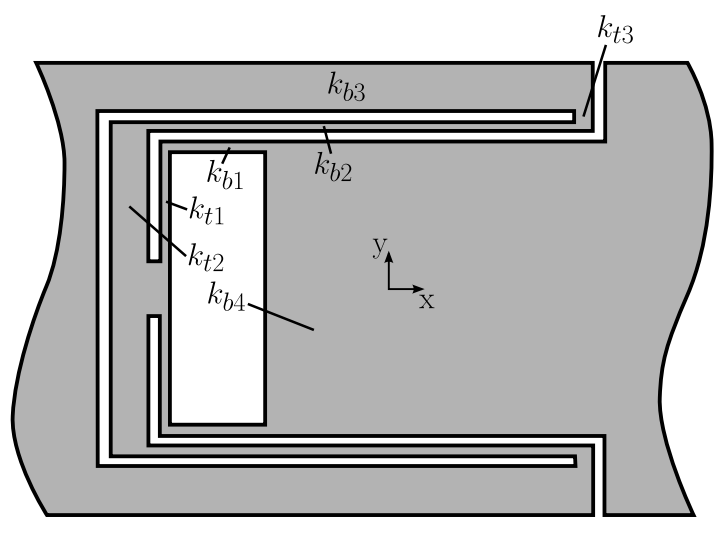

(a) The I-LEJ

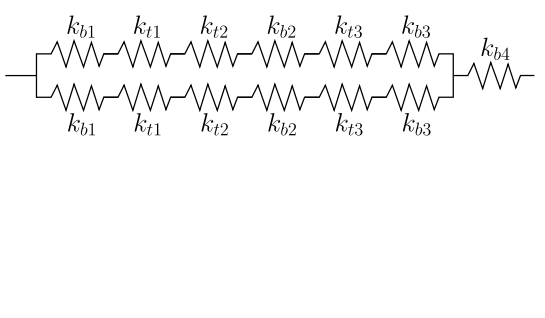

(b) Linear spring model

Figure 4: Inverted Lamina Emergent Joint (I-LEJ) and its associated linear spring model

The closed-form stiffness model shown in Equation (5) was used to optimize the ILEJ joint geometry to minimize $k_{e q, b e n d}$. The optimization constraints were based on manufacturing constraints and geometry that was constrained to a $50 \mathrm{~mm}$ long by 46 $\mathrm{mm}$ wide area. $46 \mathrm{~mm}$ was chosen as the width so that when the tension straps (which are discussed later) are added, the joint is constrained to a $50 \mathrm{~mm}$ by $50 \mathrm{~mm}$ area. The closed-form equations are well behaved and the algorithm quickly converged. In this work, the Generalized Reduced Gradient (GRG) algorithm, imlemented in OptdesX, was used for the optimization, but many different algorithms would also produce good results. The optimized dimensions are listed in Table 1.

Table 1: Dimensions of the I-LEJ optimized for minimum bending stiffness

\begin{tabular}{|c|c||c|c|}
\hline Dimension & Value $(\mathrm{mm})$ & Dimension & Value $(\mathrm{mm})$ \\
\hline$l_{b 1}$ & 8 & $l_{t 1}$ & 12 \\
\hline$w_{b 1}$ & 1 & $w_{t 1}$ & 1 \\
\hline$l_{b 2}$ & 43 & $l_{t 2}$ & 14 \\
\hline$w_{b 2}$ & 1 & $w_{t 2}$ & 4 \\
\hline$l_{b 3}$ & 47 & $l_{t 3}$ & 7 \\
\hline$w_{b 3}$ & 5 & $w_{t 3}$ & 2 \\
\hline$l_{b 4}$ & 36 & $t$ & 1 \\
\hline$w_{b 4}$ & 30 & & \\
\hline
\end{tabular}




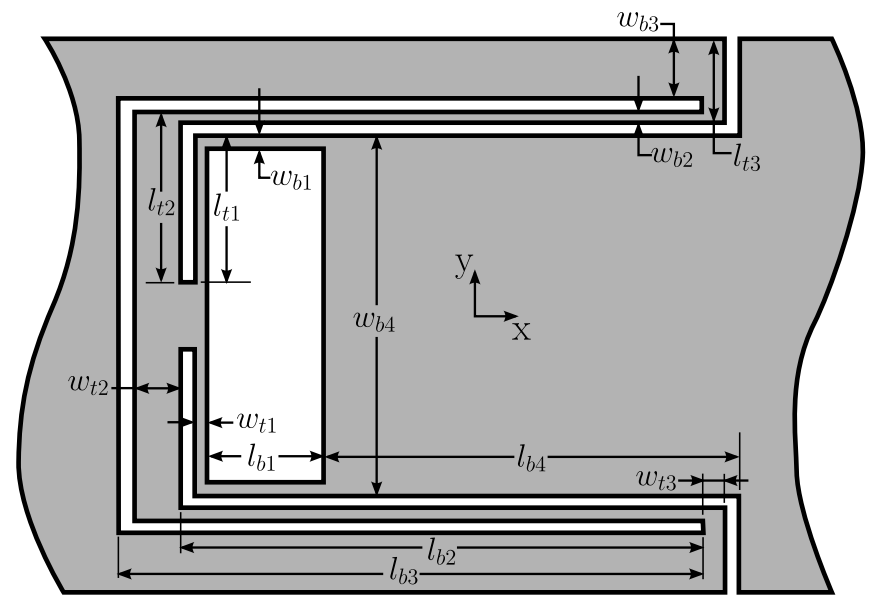

Figure 5: Dimension labels for the I-LEJ

\subsection{FEA Model}

Using the dimensions obtained from the optimization of the small-deflection equations, an FEA model of the I-LEJ was created to evaluate the joint behavior outside the range of the closed-form equations. A finite element analysis (FEA) program capable of nonlinear analysis (ANSYS@ [24]). Since the joint is symmetric, only half of it was modeled to save computation time. The joint was fixed at one end and the free end was constrained to go through a specified rotation of $30^{\circ}(0.524 \mathrm{rad})$ for bending and through an axial deflection of $0.2 \mathrm{~mm}$ in the $-x$ direction for in-plane compression. The deflected FEA bending model is shown in Figure 6 .

To compare the compressive stiffness of the I-LEJ to the LET joint benchmark, a LET joint was optimized for maximum stiffness in compression, while maintaining the same bending stiffness as the optimized I-LEJ . The total width of the optimized LET joint was $57.71 \mathrm{~mm}$. The other optimized dimensions are listed in Table 2. See Figure 2 for associated dimension labels. These dimensions were used in an FEA model of the LET joint using the same boundary conditions as those for the I-LEJ. All the FEA models for this work were created using shell (ANSYS SHELL93) elements. The I-LEJ model contained 8943 elements, and the benchmark LET joint model contained 2534 elements.

Using the FEA models, the reaction moments (in the case of bending) and forces (in the case of compression) were used to find the stiffnesses of the joints. Table 3 summarizes the results. The ideal value for the ratio of the $k_{\text {eq,bend }}$ values is 1 . With a similar bending stiffness to that of the LET joint, the I-LET is 13 times 


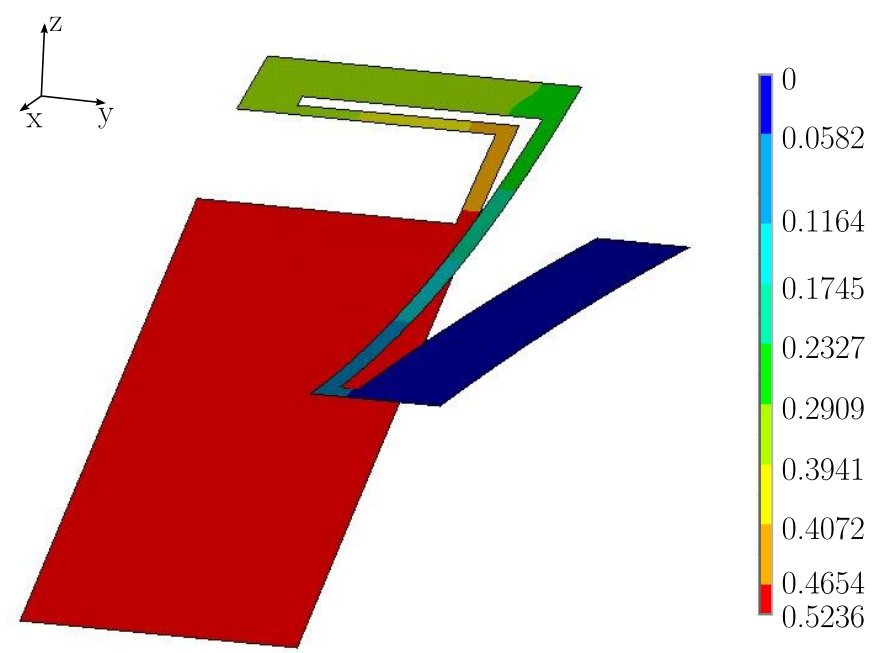

Figure 6: FEA model of the I-LEJ showing the deflected position (radians) in bending, indicating rotation about the $y$ axis

more stiff in compression. It should be noted that the closed-form model of the LET joint's compressive stiffness predicted a value $14.5 \%$ greater than that of the FEA model. This is caused by the fixed-guided boundary conditions used in the closedform model which resulted in an over-prediction of the LET joint's compressive (and tensile) stiffness. For all the LET joint geometries in this work, the closed-form model over-predicted the tensile and compressive stiffness.

The I-LEJ's ability to effectively bear compressive loads is its primary advantage. Its disadvantages include that it requires a larger area than the LET joint. Also, the joint is not suitable for high shear loading, where one end of the joint is loaded in the positive $y$ direction, and the other end in the negative $y$ direction. Another concern

Table 2: Dimensions of a benchmark LET joint optimized for a bending stiffness equivalent to the I-LEJ and maximum compressive stiffness

\begin{tabular}{|c|c|}
\hline Dimension & Value $(\mathrm{mm})$ \\
\hline$l_{b l}$ & 4 \\
\hline$w_{b l}$ & 1 \\
\hline$l_{t l}$ & 25.858 \\
\hline$w_{t l}$ & 1 \\
\hline$t$ & 1 \\
\hline
\end{tabular}




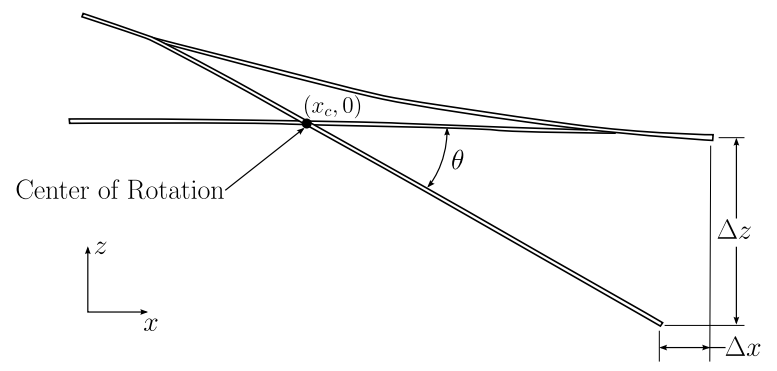

Figure 7: Side view of deflected I-LEJ

with the I-LEJ is the movement of the center of rotation throughout the motion of the joint. A side view of the deflected joint is shown in Figure 7. The position of the center of rotation is designated as $\left(x_{c}, z_{c}\right)$. Throughout the motion of the joint, $z_{c}=0 . x_{c}$ can be estimated using the following equation:

$$
x_{c}=l_{j}+\Delta x+\frac{\Delta z}{\tan \theta}
$$

where $l_{j}$ represents the total length of the joint. The $x$ position of the center of rotation is plotted against the angle of rotation in Figure 8(a). The center of rotation moves $0.418 \mathrm{~mm}$ in the $-x$ direction through the $30^{\circ}$ motion of the joint, or $0.84 \%$ of the total length of the joint. The average position of the center of rotation, $x_{c, \text { average }}$ is $18.51 \mathrm{~mm}$ from the fixed end of the joint, as measured using the coordinate system shown in Figure 8(b). The average position of the center of rotation through a deflection of $30^{\circ}$ is also shown in Figure 8(b).

Table 3: Comparison of joint stiffnesses: I-LEJ and LET joint

\begin{tabular}{|c|c|c|c|}
\cline { 2 - 4 } \multicolumn{1}{c|}{} & I-LEJ & LET Joint & Ratio \\
\hline$k_{\text {eq,bend }}(\mathrm{N} \cdot \mathrm{mm} / \mathrm{rad})$ & 2.567 & 2.593 & 0.99 \\
\hline$k_{\text {eq, comp }}(\mathrm{N} / \mathrm{m})$ & 921.2 & 70.73 & 13.02 \\
\hline
\end{tabular}




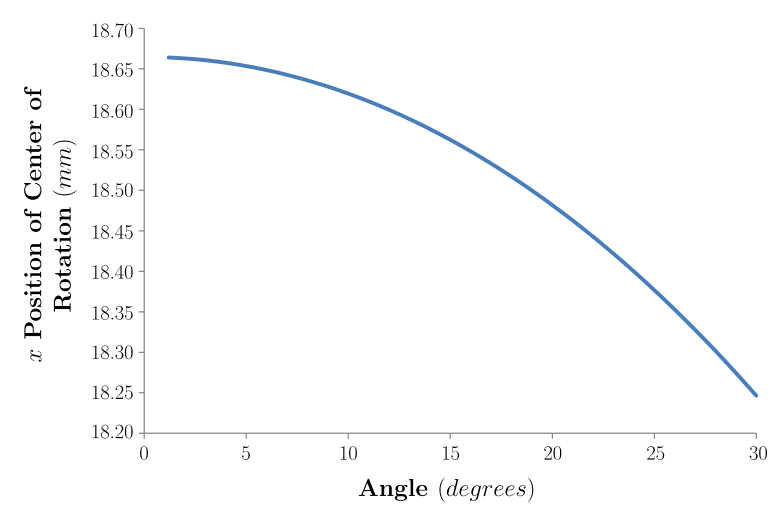

(a) $x_{c}$ plotted against angle of rotation

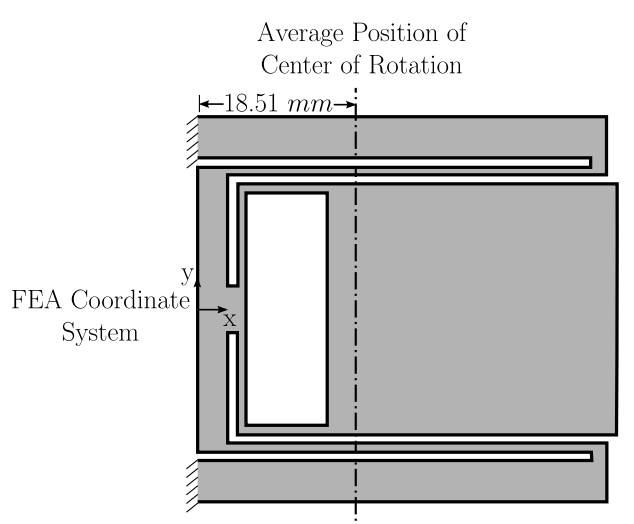

(b) Average position of the center of rotation of the I-LEJ for a $30^{\circ}$ rotation

Figure 8: Determining the center of rotation of the I-LEJ

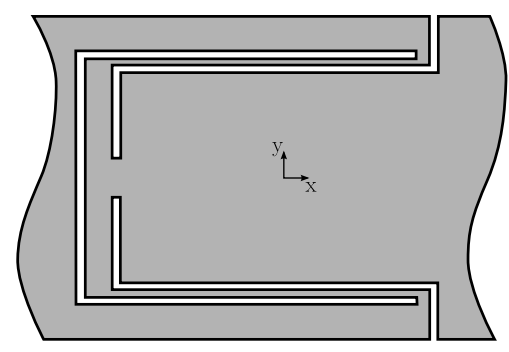

Figure 9: Alternative configuration of the I-LEJ

\subsection{Alternative Configuration}

Additional compressive stiffness can be obtained at the expense of bending flexibility using the I-LEJ can be configuration shown in Figure 9. The equations developed previously for the I-LEJ still apply, but $k_{b 1}$ and $k_{t 1}$ are no longer part of the equations. Using the same dimensions as used previously, the bending stiffness of this configuration is $3.733 \mathrm{~N} \cdot \mathrm{mm} / \mathrm{rad}, 1.45$ times larger than the bending stiffness of the baseline I-LEJ configuration and 106.5 times greater than the benchmark LET joint. The compression stiffness is $7531 \mathrm{~N} / \mathrm{m}$, or 8.17 times greater than that of the baseline configuration. Although this joint is more stiff in bending, it possesses a simpler topology, is much stiffer in compression, and the movement of the center of rotation is slightly less than that of the baseline I-LEJ configuration (the center of 
rotation moves a total of $0.326 \mathrm{~mm}$ in the $-x$ direction, or $0.65 \%$ of the joint length).

\section{The Tension Lamina Emergent Joint (T-LEJ)}

The I-LEJ is well suited for compressive loads, but is not well suited for tensile loading. The Tension Lamina Emergent Joint (T-LEJ), shown in Figure 10 with its accompanying linear spring model, is designed to bear in-plane tensile loads (in the positive $x$ direction) with very little parasitic deflection. It can be viewed as a LET joint modified with long tension "straps" that deflect easily in bending, but are rigid under tension loads. Using the same methodology as before, the T-LEJ was optimized for minimum bending stiffness. Also as before, the joint was constrained to $50 \mathrm{~mm}$ by $50 \mathrm{~mm}$ area.

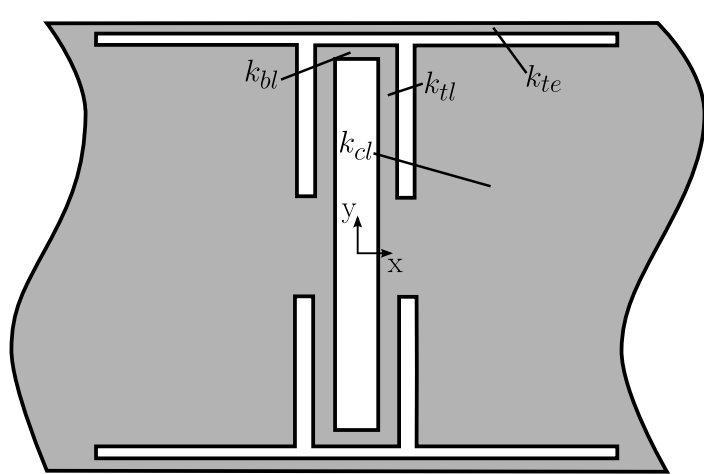

(a) The T-LEJ

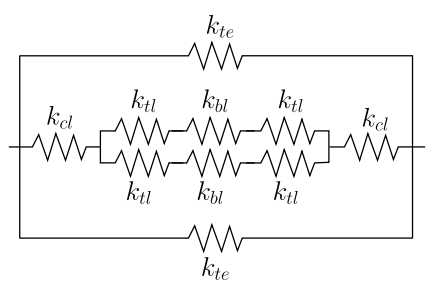

(b) Linear spring model

Figure 10: Tension Lamina Emergent Joint (T-LEJ) and its associated linear spring model

\subsection{Closed-Form Model}

Closed-form equations were developed to be used for the geometry optimization. The dimensions labels are shown in Figure 11 . The bending stiffness, $k_{e q, b e n d}$, is

$$
k_{e q, b e n d}=\frac{2 k_{b l} k_{t l} k_{c l}}{4 k_{b l} k_{t l}+k_{c l}\left(2 k_{b} l+k_{t l}\right)}+2 k_{t e}
$$

where, 


$$
\begin{aligned}
k_{b l} & =\frac{E w_{b l} t^{3}}{12 l_{b l}} \\
k_{t l} & =w_{t l} t^{3} \frac{G}{l_{t l}}\left[\frac{1}{3}-0.21 \frac{t}{w_{t l}}\left(1-\frac{t^{4}}{12 w_{t l}}\right)\right]
\end{aligned}
$$

and

$$
\begin{aligned}
k_{c l} & =\frac{E w_{c l} t^{3}}{12 l_{c l}} \\
k_{t e} & =\frac{E w_{t e} t^{3}}{12 l_{t e}}
\end{aligned}
$$

The tensile stiffness $k_{\text {eq,tens }}$, can be determined as

$$
k_{e q, t e n s}=\frac{k_{c l, a} k_{t l, a}}{2 k_{t l, a}+k_{c l, a}}+2 k_{t e, a}
$$

where

$$
\begin{aligned}
k_{c l, a} & =\frac{w_{c l} t E}{l_{c l}} \\
k_{t l, a} & =\frac{E t w_{t l}^{3}}{l_{t l}^{3}} \\
k_{t e, a} & =\frac{w_{t e} t E}{l_{t e}}
\end{aligned}
$$

Note that a given flexible element will have different stiffnesses for different loading conditions. For example, when the overall joint is in bending, the flexible element associated with $k_{t l}$ has the torsional stiffness represented by Equation (17), but when the overall joint is placed into tension, this particular element has the fixed-guided bending stiffness described by Equation (23). Different bending conditions may also result in different stiffnesses. For example, a flexible element under a pure moment will have the stiffness of the form shown in Equation (19), whereas some elements undergo fixed-guided bending under some conditions, and will have a stiffness of the form shown in Equation (23). 


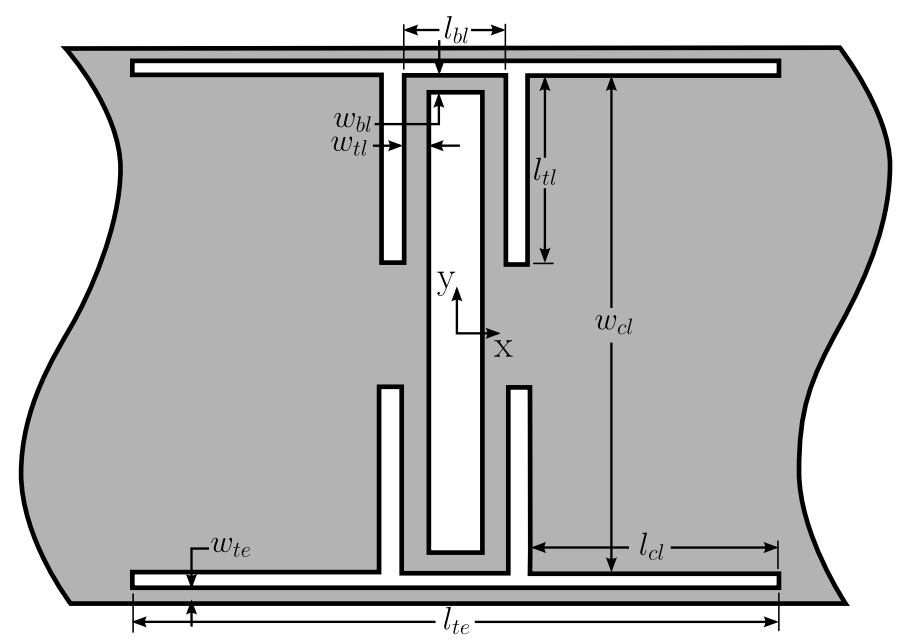

Figure 11: Dimension labels for the T-LEJ

\subsection{FEA Model}

The dimensions obtained from minimizing the bending stiffness with the given constraints are listed in Table 4. As with the I-LEJ, a benchmark LET joint was optimized for axial tensile stiffness, while maintaining the same bending stiffness as the T-LEJ. The dimensions of the optimized LET joint are listed in Table 5. Two FEA models were created for the T-LEJ: one for bending and one for tension. In Figure 12, it can be seen that the FEA model is made up of two separate entities (the main body of the joint, and the tension strap), with only boundary conditions coupling the two entities. The bending model used additional beam elements to properly couple the free ends of the two entities. These beam elements were made sufficiently rigid so that they did not significantly contribute to the deflection of the joint. The FEA model used for tension did not require the additional elements. The bending model used 5353 elements, the tension model used 5250 elements and the benchmark LET joint used 946 elements. As with the I-LEJ, the FEA model of the T-LEJ was fixed at one end and displaced $30^{\circ}$ about the $y$ axis on the other to determine the bending stiffness, and $0.2 \mathrm{~mm}$ in the $x$ to determine the tensile stiffness.

An image of the FEA bending model of the T-LEJ in its deflected position is shown in Figure 12. The results of the FEA modeling are summarized in Table 6. It can be seen in Figure 12 that the torsion members of the joint are not being deflected by pure torsion, which results in a nonlinear moment/deflection curve, which is shown in Figure 13. A trend line is also shown in this plot, with an $R^{2}$ value of 0.9967 , 


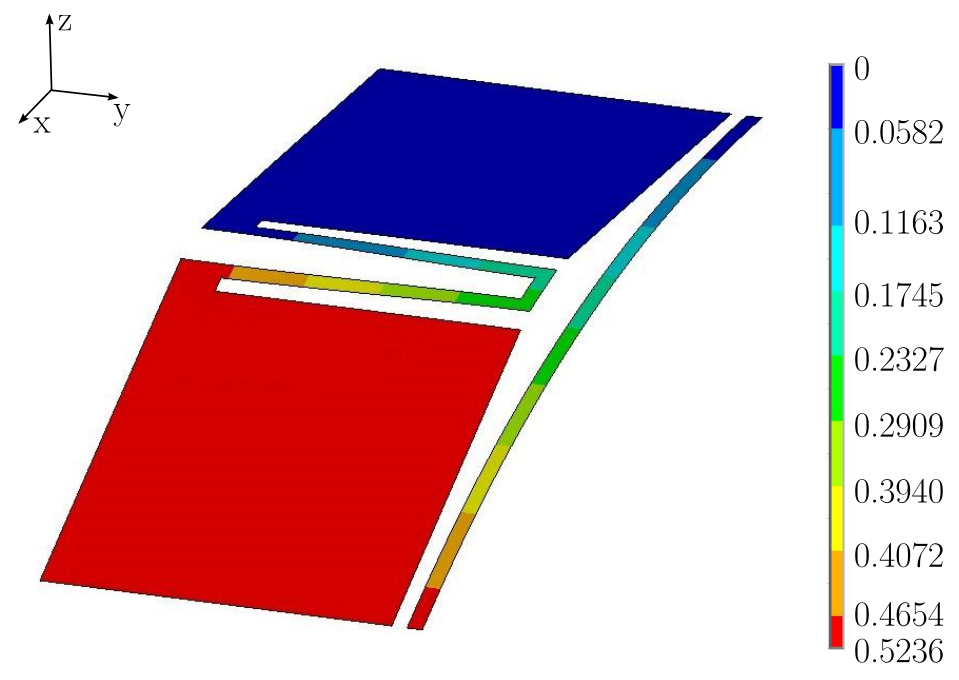

Figure 12: FEA model of the T-LEJ showing the deflected position (radians) in bending, indicating rotation about the $y$ axis

indicating that the curve is nearly linear. The value reported for the finite element $k_{e q, b e n d}$ in Table 6 is the slope of the trend line.

As shown in Table 6, the T-LEJ is 21 times stiffer to an in-plane tensile load than the benchmark LET joint with the same bending stiffness. The T-LEJ takes up more area than the LET joint, and has a slightly more complex topology, although it is still simple. It does not effectively carry compressive loads due to buckling of the tension straps. Because the joint is symmetric about the $y$ axis, determining the center of rotation of the joint can be easily determined by visual inspection. The center of rotation does move slightly throughout the bending motion of the joint as shown in Figure 14. The center of rotation moves a total of $0.57 \mathrm{~mm}$ for a $30^{\circ}$ rotation of the

Table 4: Dimensions of the T-LEJ optimized for minimum bending stiffness

\begin{tabular}{|c|c||c|c|}
\hline Dimension & Value $(\mathrm{mm})$ & Dimension & Value $(\mathrm{mm})$ \\
\hline$l_{b l}$ & 4 & $l_{c l}$ & 22 \\
\hline$w_{b l}$ & 1 & $w_{c l}$ & 46 \\
\hline$l_{t l}$ & 20 & $l_{t e}$ & 50 \\
\hline$w_{t l}$ & 1 & $w_{t e}$ & 1 \\
\hline$t$ & 1 & & \\
\hline
\end{tabular}




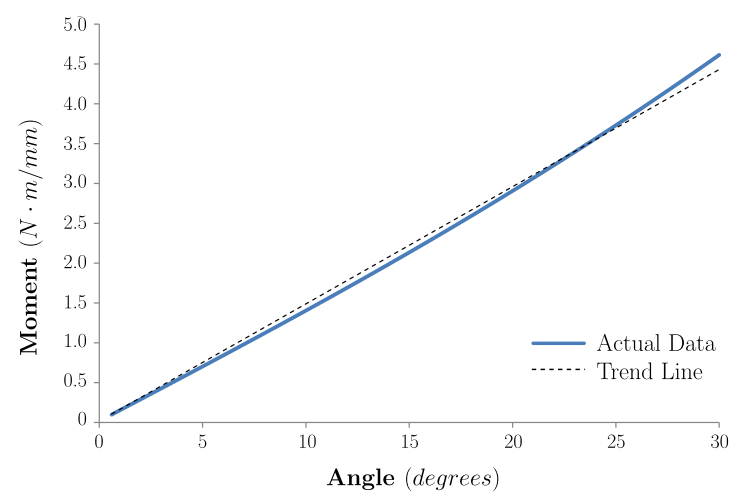

Figure 13: Moment/deflection curve for the T-LEJ

joint. This represents $1.1 \%$ of the total length of the joint.

\section{The Inverted Tension Lamina Emergent Joint (IT-LEJ)}

The Inverted Tension Lamina Emergent Joint (IT-LEJ) is a combination of the I-LEJ and T-LEJ, and is capable of effectively bearing compressive and tensile loads with minimal deflection. Closed-form equations of the IT-LEJ would not be as useful due to the complexity resulting from the interactions of the different joint components. Although closed-form equations are not available as design tools, nonlinear finite element analysis provides a means for evaluating the joints' performance. A schematic of the joint is shown in Figure 15.

\subsection{FEA Model}

Using the dimensions of the previously optimized I-LEJ and T-LEJ, an FEA model of the joint was created. The dimensions are summarized in Table 7. The

Table 5: Dimensions of a LET joint optimized for a bending stiffness equivalent to the T-LEJ and maximum tensile stiffness

\begin{tabular}{|c|c|}
\hline Dimension & Value $(\mathrm{mm})$ \\
\hline$l_{b l}$ & 4 \\
\hline$w_{b l}$ & 1 \\
\hline$l_{t l}$ & 7.046 \\
\hline$w_{t l}$ & 1 \\
\hline$t$ & 1 \\
\hline
\end{tabular}




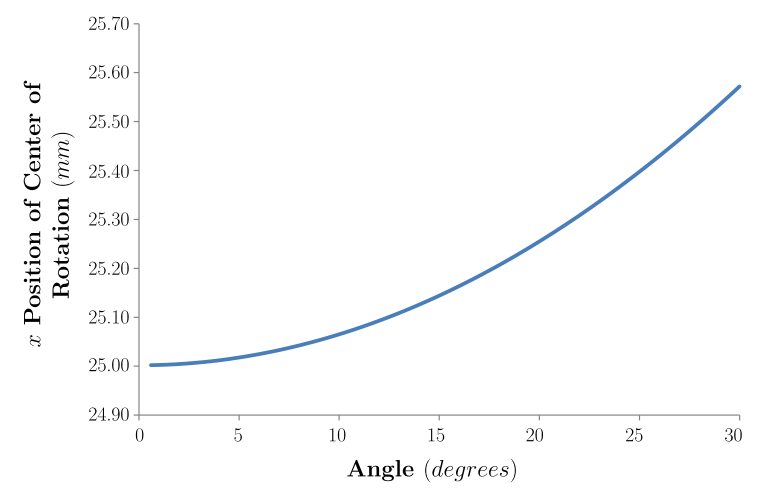

Figure 14: $x$ position of the center of rotation of the T-LEJ

labels are the same as those used for the I-LEJ, and as with the T-LEJ, $l_{t e}$ and $w_{t e}$ represent the length and width, respectively, of the tensions straps. The dimensions of the benchmark LET joint are summarized in Table 8.

The bending model included additional beam elements to ensure that the free end of the model was properly coupled; it used 9804 elements. The model used for tension and compression used 9743 elements, and the benchmark LET joint used 869 elements. The results of the FEA are summarized in Table 9. The IT-LEJ has a nonlinear moment deflection curve and its trend line has an $R^{2}$ value of 0.9712 . The slope of this line is reported as the FEA bending stiffness value in Table 9. The IT-LEJ is 6.99 times stiffer in compression then the benchmark LET joint and 7.13 times stiffer in tension.

This joint has the ability to withstand tensile and compressive loads with the trade-off of using more area than the LET joint. The movement of the center of rotation is another concern. As discussed with the T-LEJ, adding tension straps to the I-LEJ modifies the motion path of the end of the joint. The position of the center of rotation is found the same way as was used for the I-LEJ and the results are plotted in Figure 16(a). Through the $30^{\circ}$ motion of the joint, the center of rotation moves $7.2 \mathrm{~mm}$, or $14.4 \%$ of the length of the joint. The average position of the center

Table 6: Comparison of joint stiffnesses: T-LEJ and LET joint

\begin{tabular}{|c|c|c|c|}
\cline { 2 - 4 } \multicolumn{1}{c|}{} & T-LEJ & LET Joint & Ratio \\
\hline$k_{\text {eq, bend }}(\mathrm{N} \cdot \mathrm{mm} / \mathrm{rad})$ & 8.428 & 8.896 & 0.95 \\
\hline$k_{\text {eq, tens }}(\mathrm{N} / \mathrm{m})$ & 55750 & 2653 & 21.01 \\
\hline
\end{tabular}




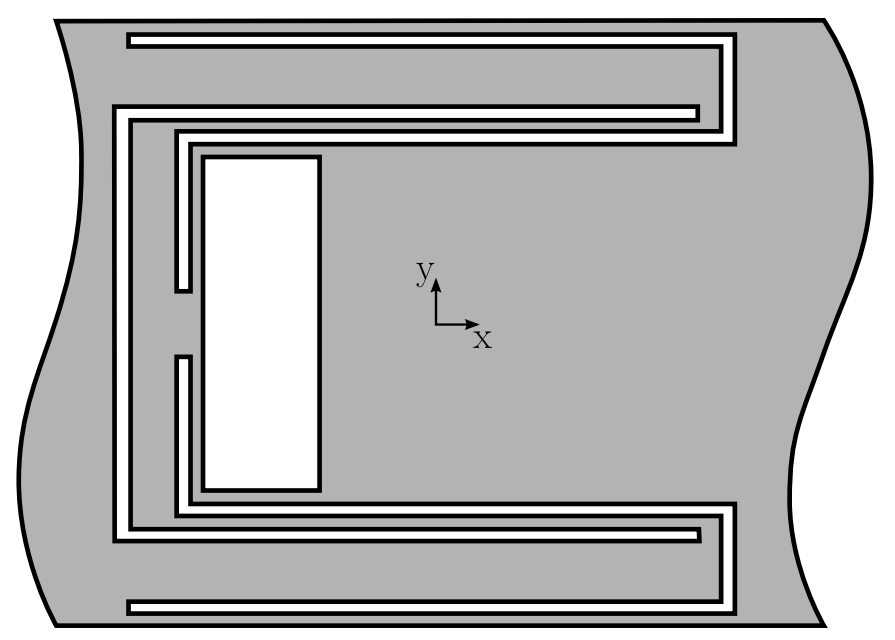

Figure 15: Schematic of the Inverted Tension Lamina Emergent Joint (IT-LEJ)

of rotation of this joint is located $21.03 \mathrm{~mm}$ from the fixed end of the joint in the FEA model's coordinate system (indicated in Figure 16(b)).

\section{Applications}

To demonstrate the joints as a part of various LEM mechanisms, a LEM fourbar was made using the IT-LEJ, as shown in Figure 1. Figure 17 shows a multilayer spherical mechanism [17, 25, 26, 27, 28, 29] made using I-LEJs. These two mechanisms illustrate the joints' compatibility with LEMs. If a mechanism like the crank-slider shown in [7], which is actuated with a compressive force, used the I-LEJ,

Table 7: Dimensions of the IT-LEJ optimized for minimum bending stiffness

\begin{tabular}{|c|c||c|c|}
\hline Dimension & Value $(\mathrm{mm})$ & Dimension & Value $(\mathrm{mm})$ \\
\hline$l_{b 1}$ & 8 & $l_{t 1}$ & 12 \\
\hline$w_{b 1}$ & 1 & $w_{t 1}$ & 1 \\
\hline$l_{b 2}$ & 43 & $l_{t 2}$ & 14 \\
\hline$w_{b 2}$ & 1 & $w_{t 2}$ & 4 \\
\hline$l_{b 3}$ & 47 & $l_{t 3}$ & 7 \\
\hline$w_{b 3}$ & 5 & $w_{t 3}$ & 2 \\
\hline$l_{b 4}$ & 36 & $l_{t e}$ & 7 \\
\hline$w_{b 4}$ & 30 & $w_{t e}$ & 2 \\
\hline$t$ & 1 & & \\
\hline
\end{tabular}




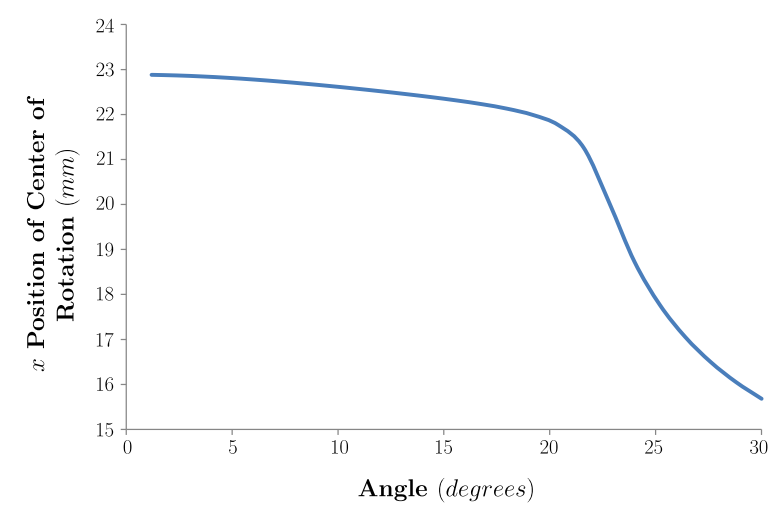

(a) $x$ position of the center of rotation of the IT-LEJ

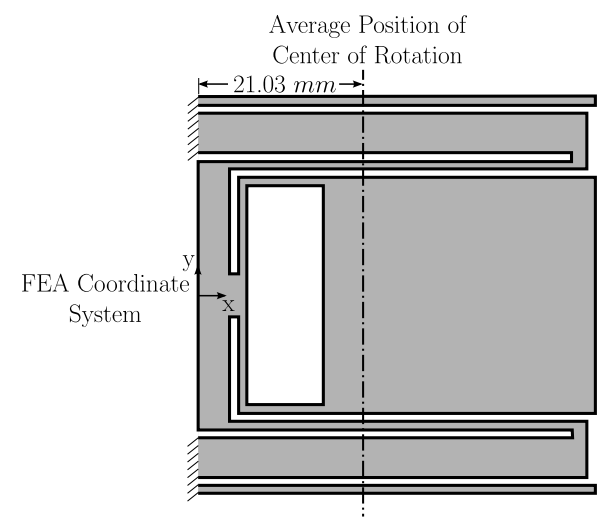

(b) Average position of the center of rotation of the IT-LEJ for a $30^{\circ}$ rotation

Figure 16: Determining the center of rotation of the IT-LEJ

the analysis could be simplified since the joints could minimize the parasitic motion in the mechanism. Other LEM applications that require tension and compression loads for actuation would benefit from the implementation of the I-LEJ, the T-LEJ, and the IT-LEJ. Several such applications are described in [25].

\section{Conclusions}

Three lamina emergent joints were introduced: the I-LEJ, the T-LEJ, and the IT-LEJ. The joints demonstrate an ability to reduce parasitic motion in LEMs when subjected to in-plane tension and/or compression loads. The applicable loading conditions for each joint, as well as important joint characteristics are summarized in Table 10, including the movement of the center of rotation, non-linearity of the

Table 8: Dimensions of the LET joint optimized for a bending stiffness equivalent to the IT-LEJ and maximum compressive and tensile stiffnesses

\begin{tabular}{|c|c|}
\hline Dimension & Value $(\mathrm{mm})$ \\
\hline$l_{b l}$ & 4.291 \\
\hline$w_{b l}$ & 1 \\
\hline$l_{t l}$ & 5 \\
\hline$w_{t l}$ & 1.131 \\
\hline$t$ & 1 \\
\hline
\end{tabular}




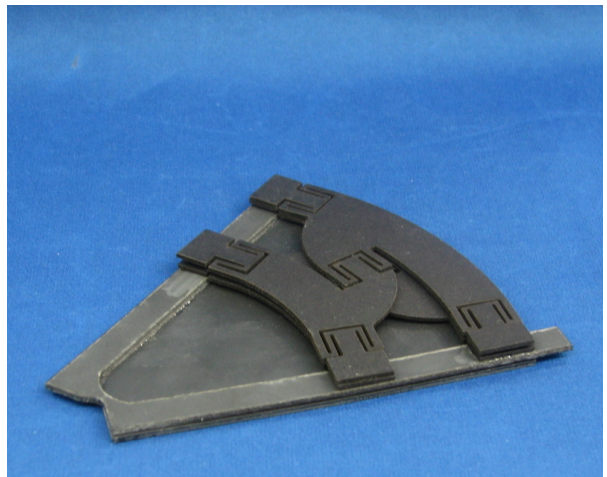

(a) Multi-layer spherical LEM in planar position

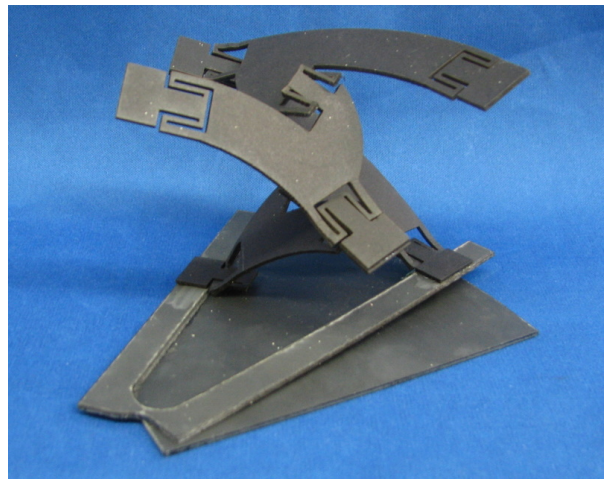

(b) Multi-layer spherical LEM in actuated position

Figure 17: Multi-layer spherical LEM with I-LEJs

moment-deflection curve, and the area required by the joint. Along with the LET joint, these joints offer a wide variety of capabilities for LEM joints.

While the joints tend to take up more area than LET joints with equivalent bending stiffnesses, they demonstrate higher stiffness in compressive and/or tensile loading loading conditions. LEMs that require tension or compression loads for actuation are examples of LEM applications [25] that would benefit from the use of these joints. Additionally, this group of joints broadens the options available to engineers developing lamina emergent mechanism applications.

\section{Acknowledgments}

This material is based upon work supported by the National Science Foundation under Grant No. CMMI-0800606. Any opinions, findings, and conclusions or recommendations expressed in this material are those of the author and do not necessarily reflect the views of the National Science Foundation. The assistance Quentin Aten is also acknowledged.

Table 9: Comparison of joint stiffnesses: IT-LEJ and LET joint

\begin{tabular}{|c|c|c|c|}
\cline { 2 - 4 } \multicolumn{1}{c|}{} & IT-LEJ & LET Joint & Ratio \\
\hline$k_{\text {eq,bend }}(\mathrm{N} \cdot \mathrm{mm} / \mathrm{rad})$ & 13260 & 14395 & 0.92 \\
\hline$k_{\text {eq, comp }}(\mathrm{N} / \mathrm{m})$ & 54960 & 7865 & 6.99 \\
\hline$k_{\text {eq, tens }}(\mathrm{N} / \mathrm{m})$ & 56690 & 7945 & 7.13 \\
\hline
\end{tabular}


Table 10: Summary of relative joint performance

\begin{tabular}{|c|c|c|c|c|}
\cline { 2 - 5 } \multicolumn{1}{c|}{} & \multicolumn{2}{c|}{ Relative Off-axis Stiffness } & \multicolumn{2}{c|}{ Performance Considerations } \\
\cline { 2 - 5 } \multicolumn{1}{c|}{} & Compression & Tension & $\begin{array}{c}\text { Non-linear Moment- } \\
\text { Deflection Curve }\end{array}$ & Area \\
\hline LET & Benchmark & Benchmark & No & Benchmark \\
\hline I-LEJ & Better & Worse & No & Worse \\
\hline T-LEJ & Same & Better & Yes & Worse \\
\hline IT-LEJ & Better & Better & Yes & Worse \\
\hline
\end{tabular}




\section{References}

[1] J. O. Jacobsen, B. G. Winder, L. L. Howell, S. P. Magleby, Lamina emergent mechanisms and their basic elements, Journal of Mechanisms and Robotics 2 (2010) 1-9.

[2] B. G. Winder, S. P. Magleby, L. L. Howell, A study of joints suitable for lamina emergent mechanisms, in: 2008 ASME International Design Engineering Technical Conferences and Computers and Information in Engineering Conference, DETC 2008, August 3, 2008 - August 6, 2008, volume 2, ASME, New York City, NY, United states, 2009, pp. 339-349.

[3] N. B. Albrechtsen, S. P. Magleby, L. L. Howell, Identifying potential applications for lamina emergent mechansism using techonology push product development, in: 34th Mechanisms and Robotics Conference, presented at - 2010 ASME International Design Engineering Technical Conferences and Computers and Information in Engineering Conference, IDETC/CIE2010, August 15, 2010 - August 18, 2010.

[4] S. Kota, Compliant systems using monolithic mechanisms, Smart Materials Bulletin 2001 (2001) 7-10.

[5] L. L. Howell, Compliant Mechanisms, Wiley-Interscience, 2001.

[6] J. O. Jacobsen, G. Chen, L. L. Howell, S. P. Magleby, Lamina emergent torsional (LET) joint, Mechanism and Machine Theory 44 (2009) 2098-2109.

[7] Q. T. Aten, S. A. Zirbel, B. D. Jensen, L. L. Howell, A numerical method for position analysis of compliant mechanisms with more degrees of freedom than inputs, Journal of Mechanical Design 133 (2011) 061009-9.

[8] B. P. Trease, Y. Moon, S. Kota, Design of Large-Displacement compliant joints, Journal of Mechanical Design 127 (2005) 788-798.

[9] D. B. Ferrell, Y. F. Isaac, S. P. Magleby, L. L. Howell, Development of criteria for lamina emergent mechanism flexures with specific application to metals, Journal of Mechanical Design 133 (2011) 031009-9.

[10] W. Yao, F. Cannella, J. S. Dai, Automatic folding of cartons using a reconfigurable robotic system, Robotics and Computer-Integrated Manufacturing 27 (2011) 604-613. 
[11] H. Zhao, S. Bi, J. Yu, Nonlinear deformation behavior of a beam-based flexural pivot with monolithic arrangement, Precision Engineering 35 (2011) 369-382.

[12] X. Pei, J. Yu, G. Zong, S. Bi, H. Su, The modeling of cartwheel flexural hinges, Mechanism and Machine Theory 44 (2009) 1900-1909.

[13] J. S. Dai, J. R. Jones, Mobility in metamorphic mechanisms of Foldable/Erectable kinds, Journal of Mechanical Design 121 (1999) 375-382.

[14] E. Pernette, S. Henein, I. Magnani, R. Clavel, Design of parallel robots in microrobotics, Robotica 15 (1997) 417420.

[15] R. J. Wood, S. Avadhanula, R. Sahai, E. Steltz, R. S. Fearing, Microrobot design using fiber reinforced composites, Journal of Mechanical Design 130 (2008) 052304-11.

[16] M. Callegari, A. Cammarata, A. Gabrielli, M. Ruggiu, R. Sinatra, Analysis and design of a spherical micromechanism with flexure hinges, Journal of Mechanical Design 131 (2009) 051003-11.

[17] S. E. Wilding, L. L. Howell, S. P. Magleby, Spherical lamina emergent mechanisms, Mechanism and Machine Theory 49 (2012) 187-197.

[18] J. B. Hopkins, M. L. Culpepper, Synthesis of precision serial flexure systems using freedom and constraint topologies (FACT), Precision Engineering 35 (2011) 638-649.

[19] J. B. Hopkins, M. L. Culpepper, Synthesis of multi-degree of freedom, parallel flexure system concepts via freedom and constraint topology (FACT) part i: Principles, Precision Engineering 34 (2010) 259-270.

[20] N. Lobontiu, Compliant mechanisms: design of flexure hinges, CRC Press, 2003.

[21] S. T. Smith, Flexures: elements of elastic mechanisms, CRC Press, 2000.

[22] A. E. Guerinot, S. P. Magleby, L. L. Howell, R. H. Todd, Compliant joint design principles for high compressive load situations, Journal of Mechanical Design 127 (2005) 774-781.

[23] R. J. Roark, W. C. Young, Formulas for stress and strain, McGraw-Hill, New York, 5th edition, 1975.

[24] ANSYS Inc, ANSYS@, 2011. 
[25] P. S. Gollnick, S. P. Magleby, L. L. Howell, An introduction to multilayer lamina emergent mechanisms, Journal of Mechanical Design 133 (2011) 081006-11.

[26] C. H. Chiang, Kinematics of Spherical Mechanisms, Cambridge University Press, 1988.

[27] J. J. Cervantes-Sánchez, H. I. Medellín-Castillo, A robust classification scheme for spherical 4R linkages, Mechanism and Machine Theory 37 (2002) 1145-1163.

[28] H. I. Medellín-Castillo, J. J. Cervantes-Sánchez, An improved mobility analysis for spherical 4R linkages, Mechanism and Machine Theory 40 (2005) 931-947.

[29] C. W. Wampler, Displacement analysis of spherical mechanisms having three or fewer loops, Journal of Mechanical Design 126 (2004) 93-100. 
Table 1: Dimensions of the I-LEJ optimized for minimum bending stiffness

Table 2: Dimensions of a benchmark LET joint optimized for a bending stiffness equivalent to the I-LEJ and maximum compressive stiffness

Table 3: Comparison of joint stiffnesses: I-LEJ and LET joint

Table 4: Dimensions of the T-LEJ optimized for minimum bending stiffness

Table 5: Dimensions of a LET joint optimized for a bending stiffness equivalent to the T-LEJ and maximum tensile stiffness

Table 6: Comparison of joint stiffnesses: T-LEJ and LET joint

Table 7: Dimensions of the IT-LEJ optimized for minimum bending stiffness

Table 8: Dimensions of the LET joint optimized for a bending stiffness equivalent to the IT-LEJ and maximum compressive and tensile stiffnesses

Table 9: Comparison of joint stiffnesses: IT-LEJ and LET joints

Table 10: Summary of relative joint performance

Figure 1a: 4R linkage in planar position

Figure 1b: 4R linkage in actuated position

Figure 1: 4R linkage with INveted Tension Lamina Emergent Joints (IT-LEJ)

Figure 2a: The LET joint

Figure 2b: Spring model of the LET joint

Figure 2: Lamina Emergent Torsional (LET) joint and its associated spring model

Figure3a: LET joint loaded in compression

Figure3b: LET joint loaded in tension

Figure3: Examples of parasitic motion in the LET joint

Figure 4a: The I-LEJ

Figure 4b: Linear spring model

Figure 4: Inverted Lamina Emergent Joint (I-LEJ) and its associated linear spring model

Figure 5: Dimension labels for the I-LEJ

Figure 6: FEA model of the I-LEJ showing the deflected position (radians) in bending, indicating rotation about the $y$ axis

Figure 7: Side view of deflected I-LEJ

Figure 8a: $x_{c}$ plotted against angle of rotation

Figure 8b: Average position of the center of rotation of the I-LEJ for a $30^{\circ}$ rotation

Figure 8: Determining the center of rotation of the I-LEJ

Figure 9: Alternative configuration of the I-LEJ

Figure 10a: The T-LEJ

Figure 10b: Linear spring model 
Figure 10: Tension Lamina Emergent Joint (T-LEJ) and its associated linear spring model

Figure 11: Dimension labels for the T-LEJ

Figure 12: FEA model of the T-LEJ showing the deflected position (radians) in bending, indicating rotation about the $y$ axis

Figure 13: Moment/deflection curve for the T-LEJ

Figure 14: $x$ position of the center of rotation of the T-LEJ

Figure 15: Schematic of the Inverted Tension Lamina Emergent Joint (IT-LEJ)

Figure 16a: $x$ position of the center of rotation of the IT-LEJ

Figure 16b: Average position of the center of rotation of the IT-LEJ for a $30^{\circ}$ rotation

Figure 16: Determining the center of rotation of the IT-LEJ

Figure 17a: Multi-layer spherical LEM in planar position

Figure 17b: Multi-layer spherical LEM in actuated position

Figure 17: Multi-layer spherical LEM with I-LEJs

\begin{tabular}{|c|c||c|c|}
\hline Dimension & Value $(\mathrm{mm})$ & Dimension & Value $(\mathrm{mm})$ \\
\hline$l_{b 1}$ & 8 & $l_{t 1}$ & 12 \\
\hline$w_{b 1}$ & 1 & $w_{t 1}$ & 1 \\
\hline$l_{b 2}$ & 43 & $l_{t 2}$ & 14 \\
\hline$w_{b 2}$ & 1 & $w_{t 2}$ & 4 \\
\hline$l_{b 3}$ & 47 & $l_{t 3}$ & 7 \\
\hline$w_{b 3}$ & 5 & $w_{t 3}$ & 2 \\
\hline$l_{b 4}$ & 36 & $t$ & 1 \\
\hline$w_{b 4}$ & 30 & & \\
\hline
\end{tabular}

\begin{tabular}{|c|c|}
\hline Dimension & Value $(\mathrm{mm})$ \\
\hline$l_{b l}$ & 4 \\
\hline$w_{b l}$ & 1 \\
\hline$l_{t l}$ & 25.858 \\
\hline$w_{t l}$ & 1 \\
\hline$t$ & 1 \\
\hline
\end{tabular}

\begin{tabular}{|c|c|c|c|}
\cline { 2 - 4 } \multicolumn{1}{c|}{} & I-LEJ & LET Joint & Ratio \\
\hline$k_{\text {eq,bend }}(\mathrm{N} \cdot \mathrm{mm} / \mathrm{rad})$ & 2.567 & 2.593 & 0.99 \\
\hline$k_{\text {eq, } \mathrm{comp}}(\mathrm{N} / \mathrm{m})$ & 921.2 & 70.73 & 13.02 \\
\hline
\end{tabular}




\begin{tabular}{|c|c||c|c|}
\hline Dimension & Value $(\mathrm{mm})$ & Dimension & Value $(\mathrm{mm})$ \\
\hline$l_{b l}$ & 4 & $l_{c l}$ & 22 \\
\hline$w_{b l}$ & 1 & $w_{c l}$ & 46 \\
\hline$l_{t l}$ & 20 & $l_{t e}$ & 50 \\
\hline$w_{t l}$ & 1 & $w_{t e}$ & 1 \\
\hline$t$ & 1 & & \\
\hline
\end{tabular}

\begin{tabular}{|c|c|}
\hline Dimension & Value $(\mathrm{mm})$ \\
\hline$l_{b l}$ & 4 \\
\hline$w_{b l}$ & 1 \\
\hline$l_{t l}$ & 7.046 \\
\hline$w_{t l}$ & 1 \\
\hline$t$ & 1 \\
\hline
\end{tabular}

\begin{tabular}{|c|c|c|c|}
\cline { 2 - 4 } \multicolumn{1}{c|}{} & T-LEJ & LET Joint & Ratio \\
\hline$k_{\text {eq, bend }}(\mathrm{N} \cdot \mathrm{mm} / \mathrm{rad})$ & 8.428 & 8.896 & 0.95 \\
\hline$k_{\text {eq, tens }}(\mathrm{N} / \mathrm{m})$ & 55750 & 2653 & 21.01 \\
\hline
\end{tabular}

\begin{tabular}{|c|c||c|c|}
\hline Dimension & Value $(\mathrm{mm})$ & Dimension & Value $(\mathrm{mm})$ \\
\hline$l_{b 1}$ & 8 & $l_{t 1}$ & 12 \\
\hline$w_{b 1}$ & 1 & $w_{t 1}$ & 1 \\
\hline$l_{b 2}$ & 43 & $l_{t 2}$ & 14 \\
\hline$w_{b 2}$ & 1 & $w_{t 2}$ & 4 \\
\hline$l_{b 3}$ & 47 & $l_{t 3}$ & 7 \\
\hline$w_{b 3}$ & 5 & $w_{t 3}$ & 2 \\
\hline$l_{b 4}$ & 36 & $l_{t e}$ & 7 \\
\hline$w_{b 4}$ & 30 & $w_{t e}$ & 2 \\
\hline$t$ & 1 & & \\
\hline
\end{tabular}

\begin{tabular}{|c|c|}
\hline Dimension & Value $(\mathrm{mm})$ \\
\hline$l_{b l}$ & 4.291 \\
\hline$w_{b l}$ & 1 \\
\hline$l_{t l}$ & 5 \\
\hline$w_{t l}$ & 1.131 \\
\hline$t$ & 1 \\
\hline
\end{tabular}




\begin{tabular}{|c|c|c|c|}
\cline { 2 - 4 } \multicolumn{1}{c|}{} & IT-LEJ & LET Joint & Ratio \\
\hline$k_{\text {eq,bend }}(\mathrm{N} \cdot \mathrm{mm} / \mathrm{rad})$ & 13260 & 14395 & 0.92 \\
\hline$k_{\text {eq,comp }}(\mathrm{N} / \mathrm{m})$ & 54960 & 7865 & 6.99 \\
\hline$k_{\text {eq, tens }}(\mathrm{N} / \mathrm{m})$ & 56690 & 7945 & 7.13 \\
\hline
\end{tabular}

\begin{tabular}{|c|c|c|c|c|}
\cline { 2 - 5 } \multicolumn{1}{c|}{} & \multicolumn{2}{c|}{ Relative Off-axis Stiffness } & \multicolumn{2}{c|}{ Performance Considerations } \\
\cline { 2 - 5 } \multicolumn{1}{c|}{} & Compression & Tension & $\begin{array}{c}\text { Non-linear Moment- } \\
\text { Deflection Curve }\end{array}$ & Area \\
\hline LET & Benchmark & Benchmark & No & Benchmark \\
\hline I-LEJ & Better & Worse & No & Worse \\
\hline T-LEJ & Same & Better & Yes & Worse \\
\hline IT-LEJ & Better & Better & Yes & Worse \\
\hline
\end{tabular}




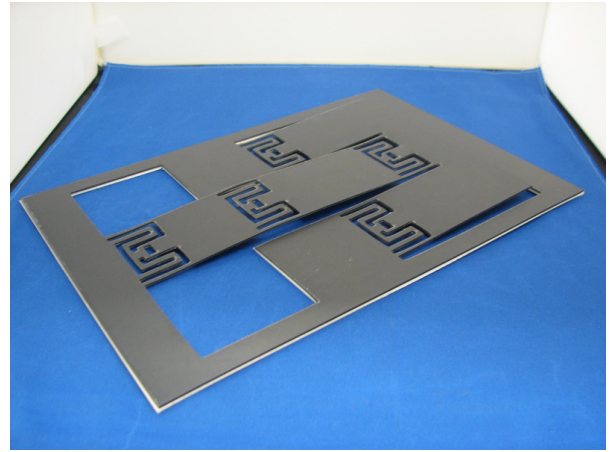

(a)

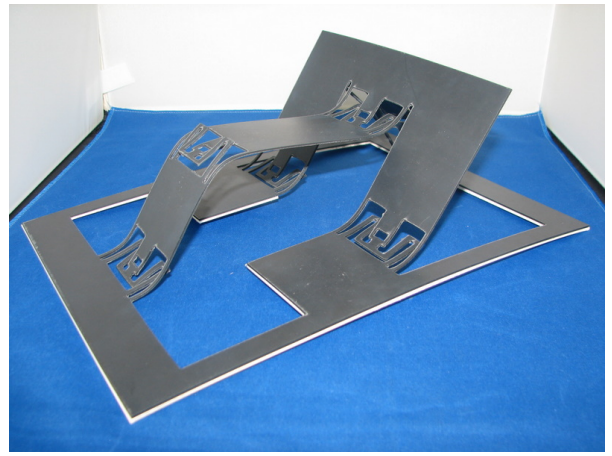

(b)

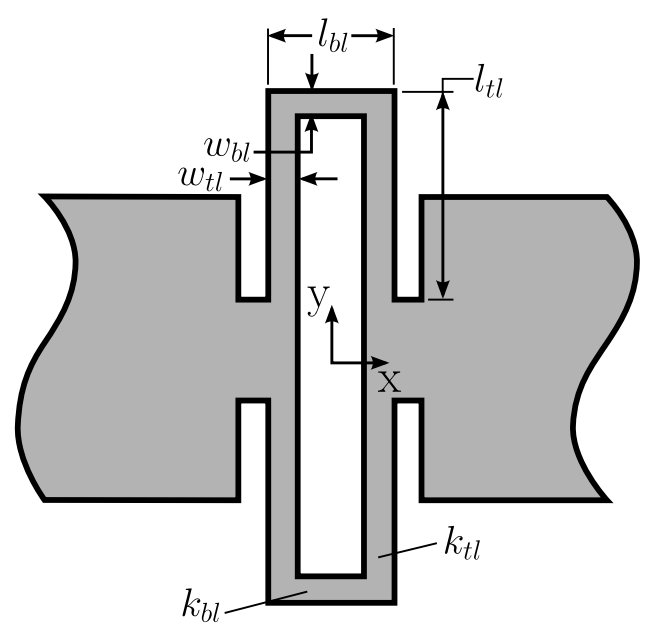

(a)

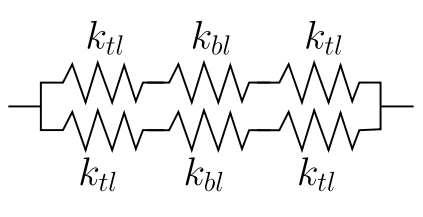

(b) 


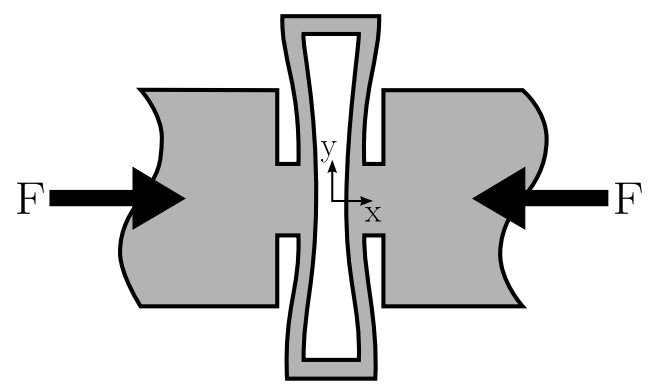

(c)

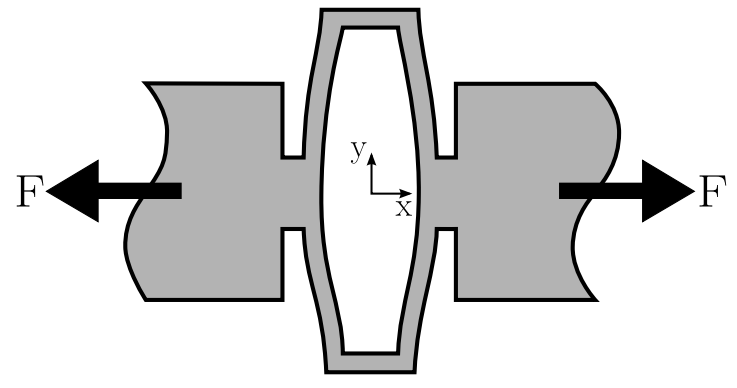

(d)

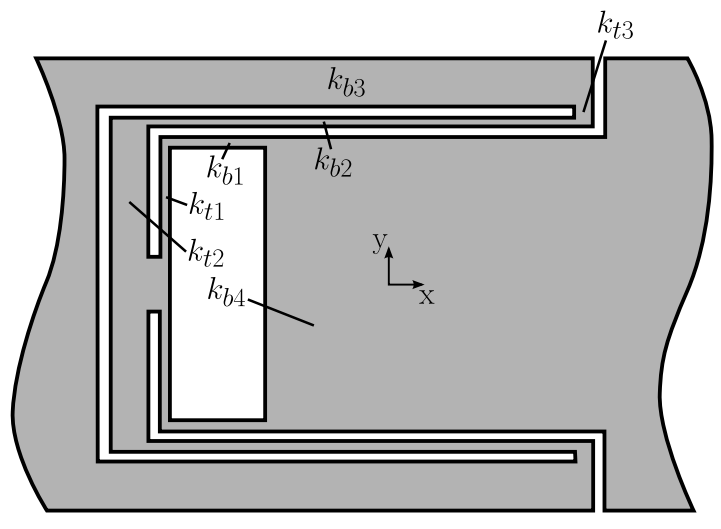

$\underbrace{k_{b 1}}_{k_{b 1}}-\underbrace{k_{t 1}}_{k_{t 1}}-\underbrace{k_{t 2}}_{k_{t 2}}-\underbrace{k_{b 2}}_{k_{b 2}}-1_{k_{t 3}}^{k_{t 3}}-\underbrace{k_{b 3}}_{k_{b 3}}$

(a)

(b) 

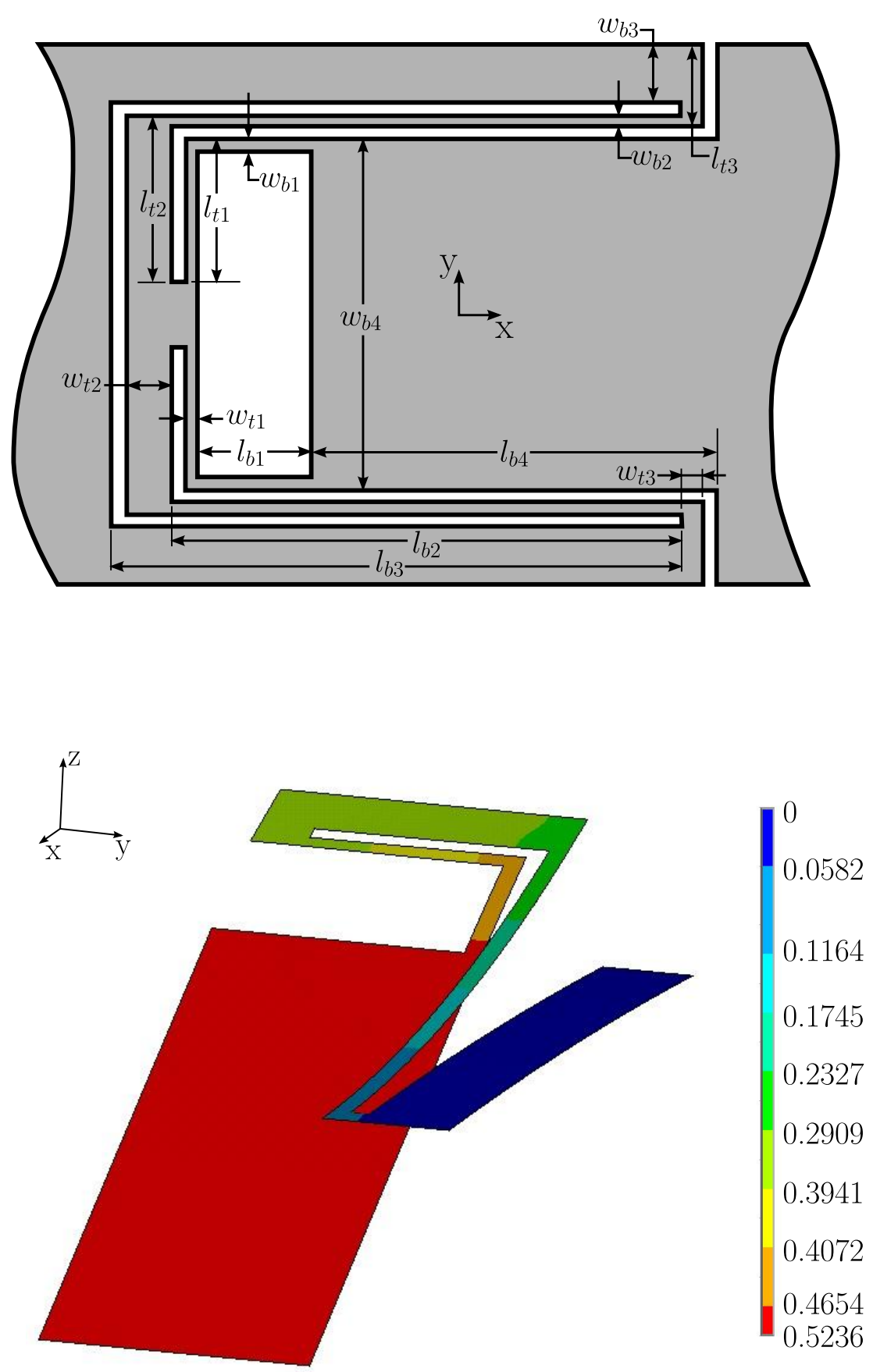


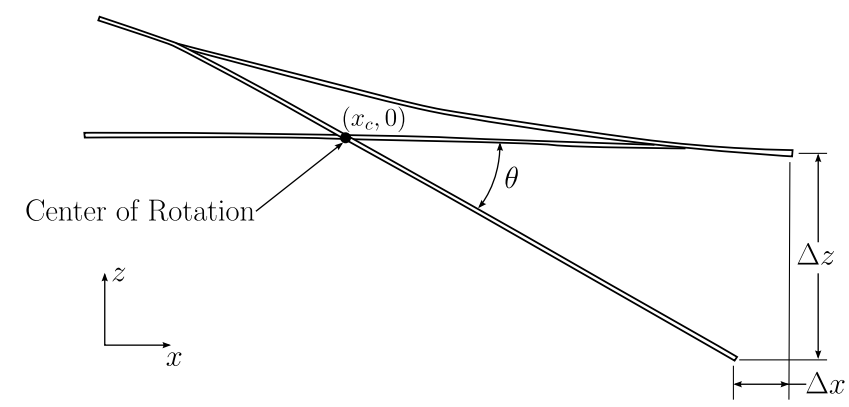




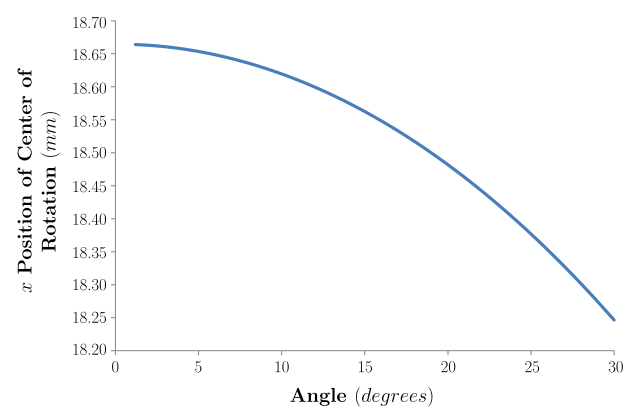

(a)

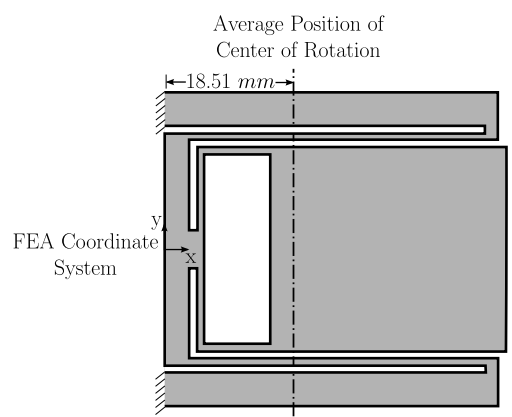

(b)

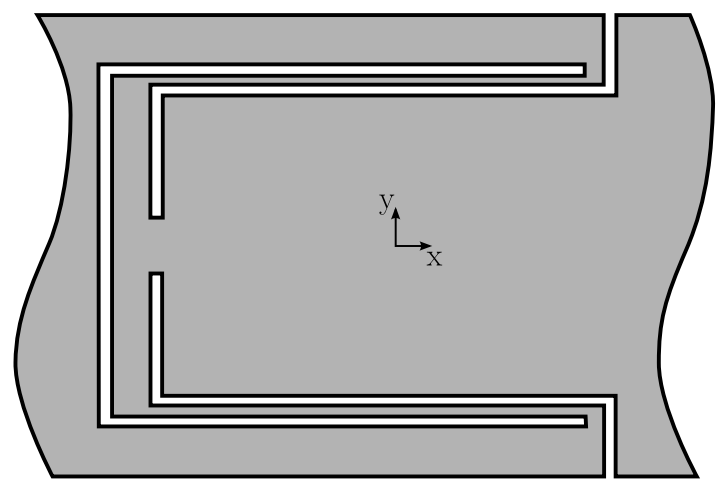



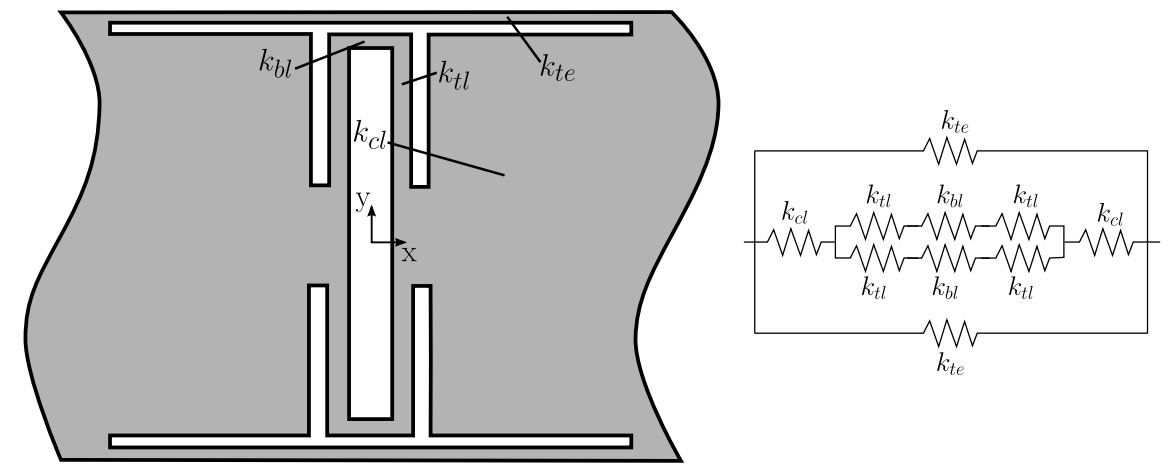

(a)

(b)

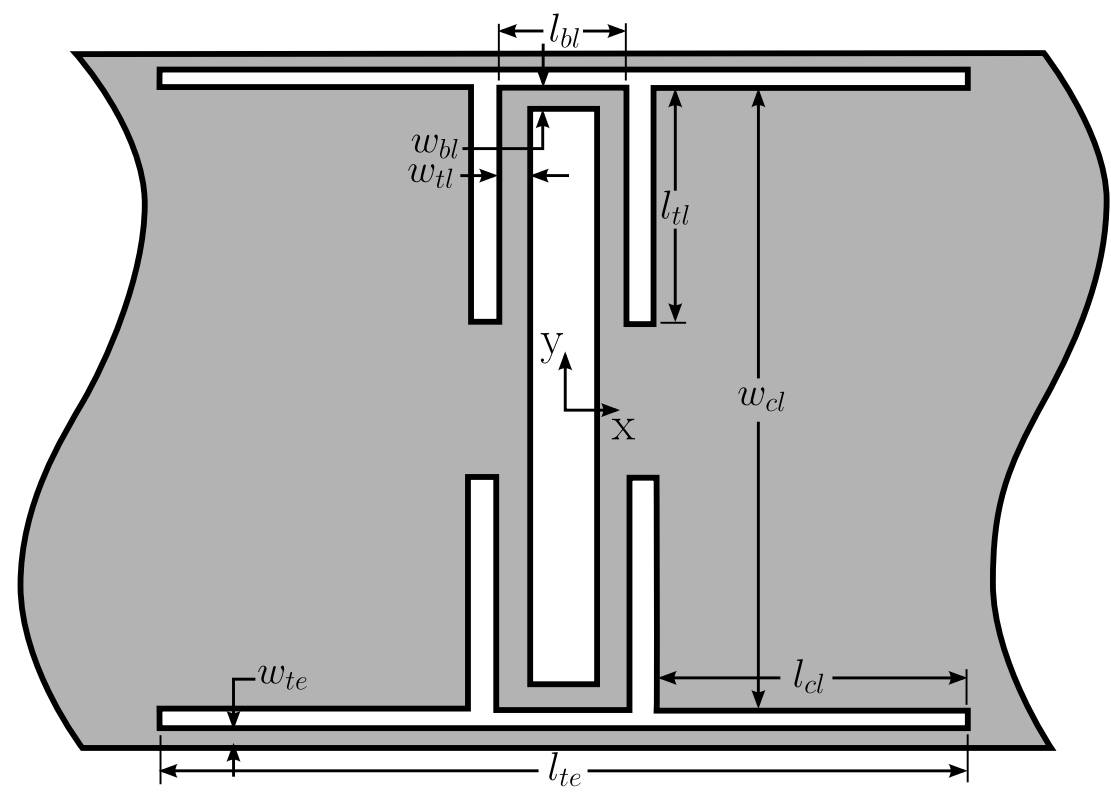



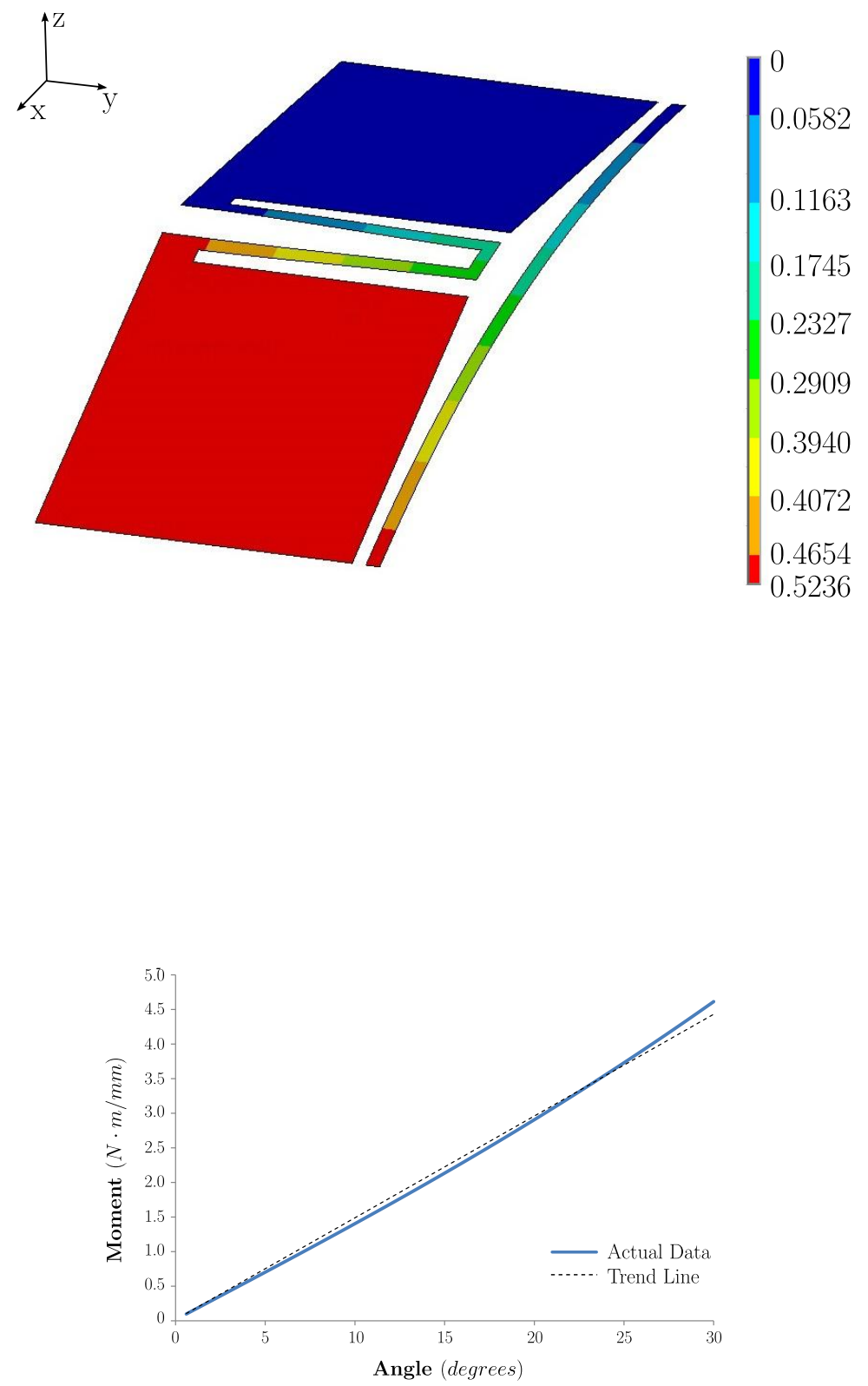

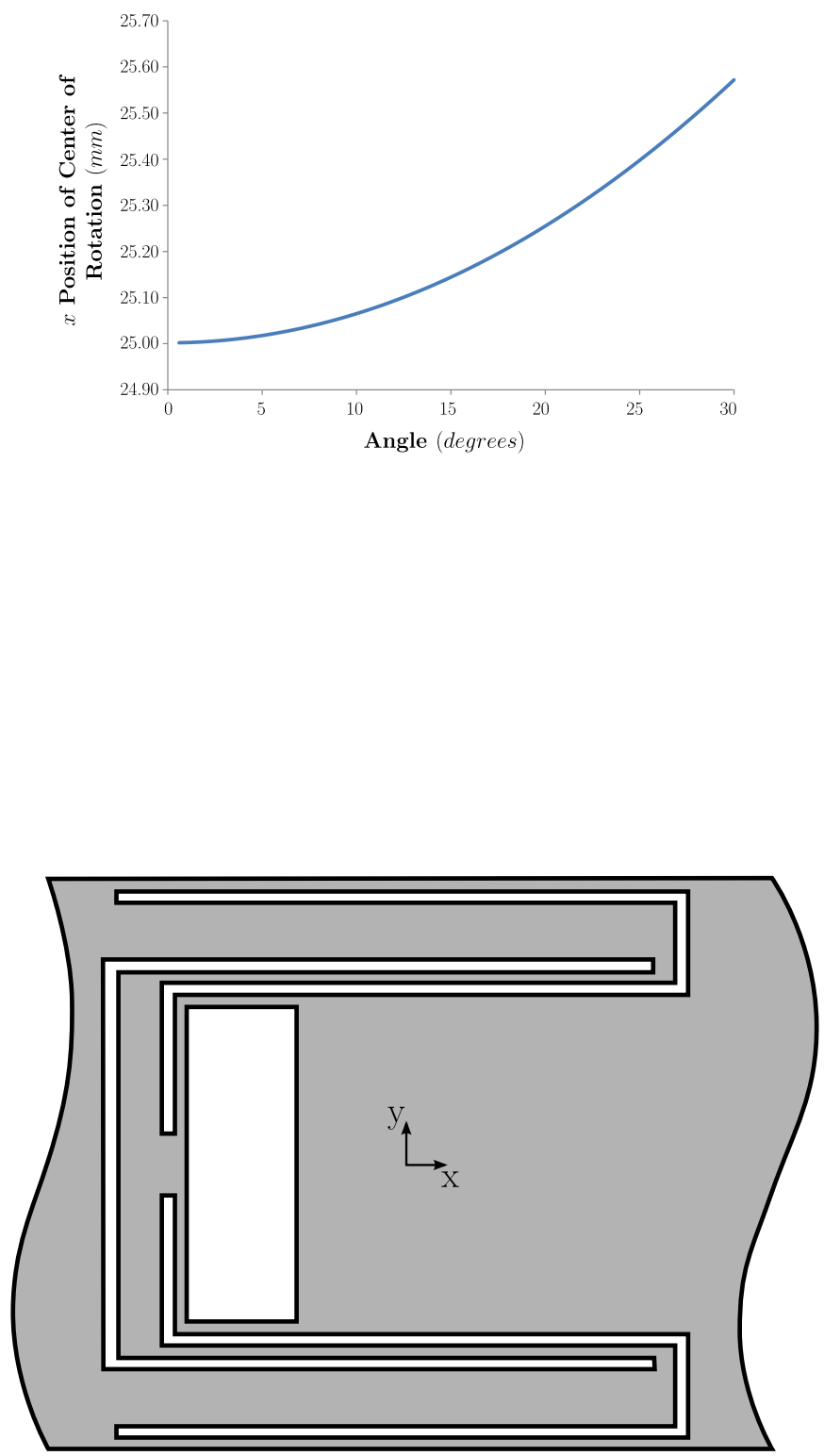


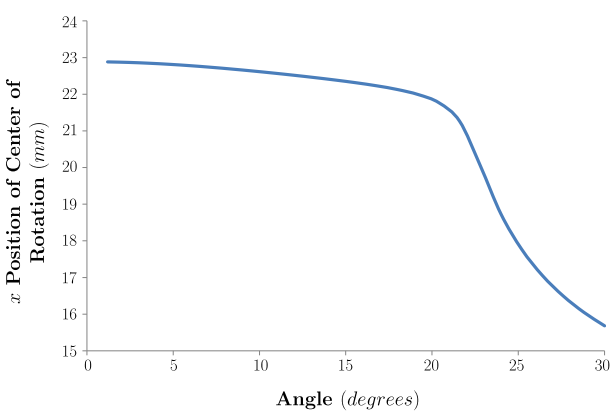

(a)

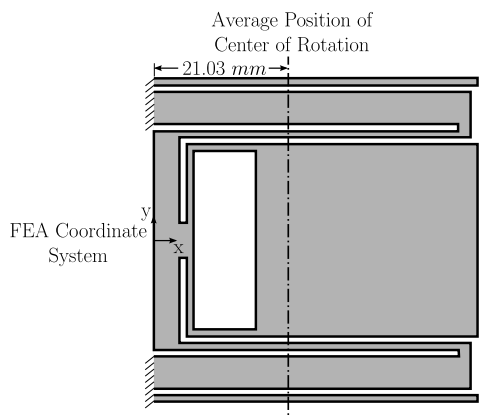

(b)

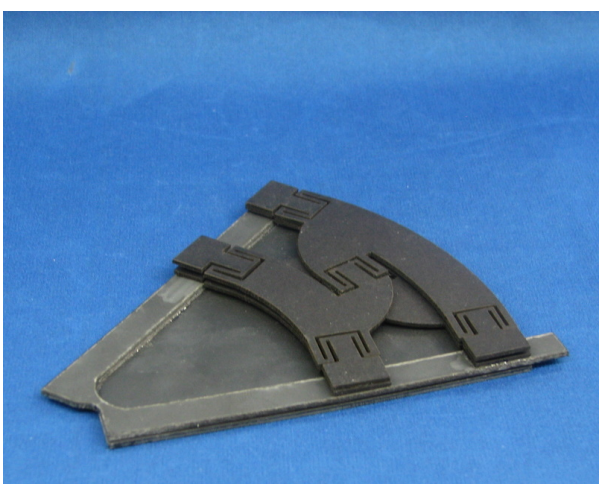

(a)

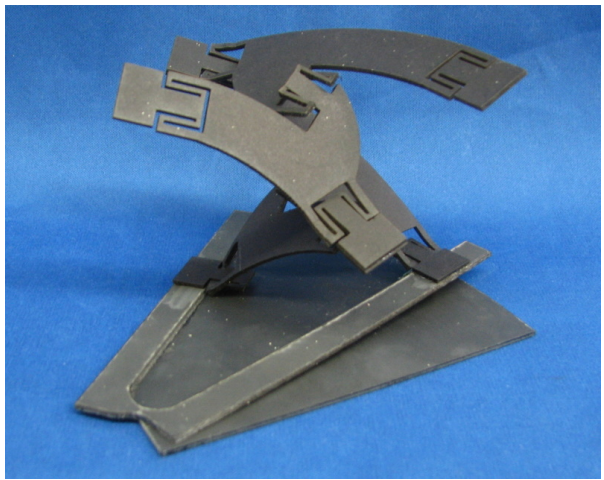

(b) 